\title{
HARMONIC CALCULUS ON P.C.F. SELF-SIMILAR SETS
}

\author{
JUN KIGAMI
}

\begin{abstract}
The main object of this paper is the Laplace operator on a class of fractals. First, we establish the concept of the renormalization of difference operators on post critically finite (p.c.f. for short) self-similar sets, which are large enough to include finitely ramified self-similar sets, and extend the results for Sierpinski gasket given in [10] to this class. Under each invariant operator for renormalization, the Laplace operator, Green function, Dirichlet form, and Neumann derivatives are explicitly constructed as the natural limits of those on finite pre-self-similar sets which approximate the p.c.f. self-similar sets. Also harmonic functions are shown to be finite dimensional, and they are characterized by the solution of an infinite system of finite difference equations.
\end{abstract}

\section{INTRODUCTION}

Mathematical analysis has recently begun on fractal sets. The pioneering works are the probabilistic approaches of Kusuoka [11] and Barlow and Perkins [2]. They have constructed and investigated Brownian motion on the Sierpinski gaskets. In their standpoint, the Laplace operator has been formulated as the infinitesimal generator of the diffusion process.

On the other hand, in [10], we have found the direct and natural definition of the Laplace operator on the Sierpinski gaskets as the limit of difference operators. In the present paper, we extend the results in [10] to a class of self-similar sets called p.c.f. self-similar sets which include the nested fractals defined by Lindstrøm [13]. Several examples of p.c.f. self-similar sets are given in the figures of $\xi 8$. The reader can find an exposition of the original ideas of this work in $\S 0$ of $[10]$.

In $\S 1$, we study some topological properties of general self-similar sets and define p.c.f. self-similar sets. Roughly speaking, p.c.f. self-similar sets are almost the same concept as "finitely ramified fractals" mainly used by physicists. We note that the Sierpinski carpet, where Barlow and Bass [1] have constructed a diffusion process, is not a p.c.f. self-similar set.

In $\S 2$, we introduce the concept of a quasi-harmonic structure on p.c.f. selfsimilar sets. It induces a sequence of the difference operators which correspond to the discrete Laplace operators. When a quasi-harmonic structure satisfies some condition for invariance, we call it a harmonic structure, and we will find

Received by the editors August 28, 1989 and, in revised form, October 29, 1990.

1980 Mathematics Subject Classification (1985 Revision). Primary 31C05, 31C20, 31C25, 39A10; Secondary 39A12, 60J99, 65P05.

Key words and phrases. Self-similar sets, harmonic structures, harmonic functions, Green function, Laplace operator, Dirichlet forms. 
explicit and simple definitions of harmonic functions, the Green function, and the Laplace operator.

In $\S 6$, we treat the Dirichlet problem of Poisson's equation and Gauss-Green's formula on p.c.f. self-similar sets. Further, we will see that the Dirichlet problem of Poisson's equation is equivalent to some kind of infinite system of finite difference equations. This fact has been pointed out by Hata-Yamaguti [6] and Yamaguti-Kigami [15] in the simplest case.

In $\S 7$, we give an explicit and simple definition of Dirichlet forms associated with regular harmonic structures. And then, the Green function turns out to be the reproducing kernel of the Dirichlet form $(\mathscr{E}, \mathscr{F})$.

As a whole, our approach establishes a kind of classical calculus on p.c.f. selfsimilar set, and it may be more convenient to the study of harmonic functions and the Laplace operator than the probabilistic approaches.

Finally we mention three related works. The first one and the second one are the probabilistic approaches on a class of self-similar sets.

Lindstrøm [13] has constructed the diffusion processes on nested fractals. The readers may refer to Example 8.5 and Remarks after Definition 1.10 and Definition 4.4.

Kusuoka [12] has given an explicit expression of Dirichlet forms on a class of self-similar sets by using products of random matrices. We also use random matrices $A_{s}$ 's obtained by quasi-harmonic structure. These random matrices are correspondent with those used by Kusuoka. We conjecture that our Dirichlet forms and the Dirichlet forms given by Kusuoka are the same in the correspondent cases.

Shima [14] and Fukushima-Shima [16] have studied the eigenvalue problem of the Laplace operator given by [10]. They apply "the decimation method" and determine the eigenvalues and eigenvectors completely. We conjecture that their method can be applied to our Laplace operator on p.c.f. self-similar sets.

In this paper, we adopt the "directory" structure in numbering the lemmas, propositions, and theorems. For example, Lemma 2.7.1 is the lemma for the proof of Lemma 2.7. Ordinarily, Lemma I.J.K is used only for the proof of Lemma or Proposition or Theorem I.J.

I would like to express my gratitude to Professors M. Fukushima and S. Kusuoka. In particular, the results on Dirichlet forms would not have been achieved without discussions with Professor M. Fukushima. I also thank Mr. A. Kameyama for the simulating discussion on p.c.f. self-similar sets.

\section{Self-Similar SETS}

In this section, we will define self-similar sets and study their fundamental properties.

First, we introduce the one-sided shift space and give some basic concepts and notations.

Definition 1.1. Let $S=\left\{s_{1}, s_{2}, \ldots, s_{N}\right\}$ be a finite set.

(1) The one-sided shift space $\Sigma(S)$ is defined by $\Sigma(S)=S^{\mathbb{N}}$.

(2) For $n \geq 0$, the collection of words consisting of $n$ symbols $W_{n}(S)$ is defined by $W_{n}(S)=S^{n}$. In particular, $W_{0}(S)=\{\varnothing\}$ where $\varnothing$ denotes the empty word.

(3) $W_{*}(S)=\bigcup_{n \geq 0} W_{n}(S)$. 
(4) $\quad \Sigma_{*}(S)=W_{*}(S) \cup \Sigma(S)$.

We denote the $i$ th symbol of $w \in \Sigma_{*}(S)$ by $w_{i}$ or $(w)_{i}$ and write $w=$ $w_{1} w_{2} w_{3} \cdots$.

It is easy to verify that $\Sigma(S)$ is compact with the metric $d_{\Sigma(S)}$ given by, for $w$ and $v \in \Sigma(S)$,

$$
d_{\Sigma(S)}(w, v)=\sum_{i=1}^{\infty} 2^{-i}\left(1-\delta_{w_{i} v_{i}}\right)
$$

where

$$
\delta_{\alpha \beta}= \begin{cases}1 & \text { if } \alpha=\beta \\ 0 & \text { otherwise }\end{cases}
$$

Definition 1.2. (1) For $w \in \Sigma_{*}(S)$,

$$
|w|= \begin{cases}n & \text { if and only if } w \in W_{n}(S), \\ \infty & \text { if } w \in \Sigma(S) .\end{cases}
$$

(2) Let $w \in \Sigma_{*}(S)$, then for $n \geq 0$,

$$
[w]_{n}= \begin{cases}w & \text { if }|w| \leq n \\ w_{1} w_{2} \cdots w_{n} & \text { otherwise }\end{cases}
$$

In particular, $[w]_{0}=\varnothing$ for all $w \in \Sigma_{*}(S)$.

(3) Let $w \in \Sigma_{*}(S)$, then

$$
\sigma w= \begin{cases}\varnothing & \text { if } w=\varnothing, \\ w_{2} w_{3} \cdots w_{n} & \text { if } w \in W_{n}(S), \\ w_{2} w_{3} w_{4} \cdots & \text { if } w \in \Sigma(S) .\end{cases}
$$

(4) Let $w$ and $v \in \Sigma_{*}(S)$, then $w \wedge v=[w]_{k}$, where $k=\max \left\{n \mid[w]_{n}=\right.$ $\left.[v]_{n}\right\}$.

(5) Let $w \in W_{*}(S)$ and $v \in \Sigma_{*}(S)$, then $w \cdot v \in \Sigma_{*}(S)$ is defined by

$$
(w \cdot v)_{i}= \begin{cases}w_{i} & \text { if } i \leq|w|, \\ v_{i-|w|} & \text { otherwise. }\end{cases}
$$

We always identify $w \in W_{*}(S)$ with $\tilde{w}: \Sigma(S) \rightarrow \Sigma(S)$ defined by $\tilde{w}(v)=$ $w \cdot v$. In particular, $\varnothing \in W_{0}(S)$ is identified with the identity map of $\Sigma(S)$.

The following definition of a self-similar structure is an abstraction of topological features from the concepts of the self-similar sets studied by Hutchinson [8] and Hata [5].

Definition 1.3. Let $K$ be a compact metric space, $S$ be a finite set, and, for each $s \in S$, let $F_{s}: K \rightarrow K$ be a continuous injection. Then $\left(K, S,\left\{F_{s}\right\}_{s \in S}\right)$ is said to be a self-similar structure on $K$ (or simply, $K$ is self-similar) if there exists a continuous surjection $\pi: \Sigma(S) \rightarrow K$ satisfying

$$
\pi \circ s=F_{s} \circ \pi \text { for every } s \in S \text {. }
$$

Further, for $w \in W_{*}(S)$, we define

$$
F_{w}=F_{w_{1}} \circ F_{w_{2}} \circ \cdots \circ F_{w_{n}},
$$

where $n=|w|$, and $K_{w}=F_{w}(K)$. In particular, $F_{\varnothing}$ is the identity map of $K$. 
The following result implies that $\pi$ is uniquely determined for a given selfsimilar structure.

Proposition 1.4. Let $\left(K, S,\left\{F_{s}\right\}_{s \in S}\right)$ be a self-similar structure on $K$. Then, for all $w \in \Sigma(S), \bigcap_{n \geq 0} K_{[w]_{n}}$ consists of a single point $\pi(w)$.

Proof. For $w \in \Sigma(S)$, we have $\bigcap_{n \geq 0}[w]_{n} \cdot \Sigma(S)=\{w\}$, and the diameter of $[w]_{n} \cdot \Sigma(S) \rightarrow 0$ as $n \rightarrow \infty$. As $\pi$ is continuous and $\pi\left([w]_{n} \cdot \Sigma(S)\right)=K_{[w]_{n}}$, we have $\{\pi(w)\}=\bigcap_{n \geq 0} K_{[w]_{n}}$.

Let $\mathscr{L}=\left(K, S,\left\{F_{s}\right\}_{s \in S}\right)$ be a self-similar structure on $K$. It is easy to see that $\pi$ is a homeomorphism if and only if $C_{K}(\mathscr{L})=\bigcup_{s, t \in S, s \neq t}\left(K_{s} \cap K_{t}\right)$ is empty. Further, if $C_{K}(\mathscr{L})=\varnothing$, then each $F_{s}$ is a branch of the inverse of a well-defined map $\pi \circ \sigma \circ \pi^{-1}: K \rightarrow K$.

Definition 1.5. Let $\mathscr{L}=\left(K, S,\left\{F_{s}\right\}_{s \in S}\right)$ be a self-similar structure on $K$. Then the critical set of $\mathscr{L}$ is defined by $C(\mathscr{L})=\pi^{-1}\left(C_{K}(\mathscr{L})\right)$, and the post critical set of $\mathscr{L}$ is defined by $P(\mathscr{L})=\bigcup_{n \geq 1} \sigma^{n}(C(\mathscr{L}))$.

Examples of self-similar structures are given in $\S 8$.

Hereafter, we discuss only one fixed self-similar structure, and so we use $\Sigma, W_{n}, P, C$, and so on instead of $\Sigma(S), W_{n}(S), P(\mathscr{L}), C(\mathscr{L})$, and so on.

Definition 1.6. Let $\left(K, S,\left\{F_{s}\right\}_{s \in S}\right)$ be a self-similar structure on $K$. Then,

(1) For $w \in W_{*}, B_{w}=F_{w}(\pi(P))$;

(2) For $m \geq 0, P^{(m)}=\bigcup_{w \in W_{m}} w P$ and $V_{m}=\pi\left(P^{(m)}\right)$;

(3) $\quad V_{*}=\bigcup_{m \geq 0} V_{m}$ and $V_{m}^{\circ}=V_{m}-V_{0}$ for $m=0,1, \ldots, *$.

Lemma 1.7. Let $K$ be a self-similar set. Then, for any $w$ and $v \in W_{n}$ with $w \neq v, K_{w} \cap K_{v}=B_{w} \cap B_{v}$.

Proof. As $F_{w \wedge v}$ is injective, we may assume $w_{1} \neq v_{1}$ without loss of generality. Then, since $K_{w} \cap K_{v} \subset C_{K}$,

$$
K_{w} \cap K_{v} \subset \pi(C \cap w \Sigma) \cap \pi(C \cap v \Sigma) .
$$

On the other hand, $C \subset \sigma^{-1}(P)=\bigcup_{s \in S} s P$. Hence, $C \cap w \Sigma \subset w P$ and $C \cap v \Sigma \subset v P$ and therefore $K_{w} \cap K_{v} \subset B_{w} \cap B_{v}$. Trivially, $K_{w} \cap K_{v} \supset B_{w} \cap B_{v}$, and thus we have proved the lemma.

Lemma 1.8. If $\pi(w) \in V_{0}$, then $w \in P$. In other words, $\pi^{-1}(\pi(P))=P$.

Proof. As $V_{0}=\pi(P)$, there exist $u \in P$ and a word $v \neq \varnothing$ such that $\pi(w)=$ $\pi(u)$ and $v \cdot u \in C$. Then, $\pi(v \cdot u)=\pi(v \cdot w)$ and so, $v \cdot w \in C$. Thus we have $w \in P$.

Corollary 1.9. For $m \geq 0$, if $\pi(w) \in V_{m}$, then $w \in P^{(m)}$. In other words, $\pi^{-1}\left(\pi\left(P^{(m)}\right)\right)=P^{(m)}$.

Proof. As $V_{m}=\bigcup_{v \in W_{m}} B_{v}, \pi(w) \in B_{v}$ for some $v \in W_{m}$. Using Lemma 1.7, $\pi(w) \in B_{v} \cap K_{[w]_{m}}=B_{v} \cap B_{[w]_{m}}$. This implies $\pi(w) \in B_{[w]_{m}}=F_{[w]_{m}}(\pi(P))$ and hence $\pi\left(\sigma^{m} w\right) \in \pi(P)$. Now using Lemma 1.8, we have $\sigma^{m} w \in P$. Thus we have

$$
[w]_{m} \cdot \sigma^{m} w=w \in[w]_{m} P \subset P^{(m)} .
$$

The last three results will underlie many arguments in this paper, and frequently, we may not mention using them.

Next, we introduce a class of canonical measures on self-similar sets. 
Definition 1.10. Let $\eta=\left(\eta_{s}\right)_{s \in S}$ satisfy

$$
\sum_{s \in S} \eta_{s}=1 \text { and } 0<\eta_{s}<1 \text { for each } s \in S \text {. }
$$

Then we denote by $\tilde{\mu}_{\eta}$ the unique Borel measure on $\Sigma$ with, for all $w \in W_{*}$,

$$
\tilde{\mu}_{\eta}(w \Sigma)=\eta_{w_{1}} \eta_{w_{2}} \cdots \eta_{w_{n}}
$$

where $n=|w|$. Further, let $K$ be a self-similar set. Then the $\eta$-self-similar measure on $K, \mu_{\eta}$ is given by $\pi_{*}\left(\tilde{\mu}_{\eta}\right)$, that is,

$$
\mu_{\eta}(A)=\tilde{\mu}_{\eta}\left(\pi^{-1}(A)\right)
$$

for all Borel sets $A \subset K$.

The following theorem explains why $\mu_{\eta}$ is called $\eta$-self-similar.

Theorem 1.11. Let $K$ be a self-similar set, and let $\eta$ satisfy (1.1). Then there exists a unique Borel measure $\mu$ on $K$ such that $\mu(K)=1$ and

$$
\mu(A)=\sum_{s \in S} \eta_{s} \mu\left(F_{s}^{-1}\left(A \cap K_{s}\right)\right)
$$

for all Borel sets $A \subset K$. The unique measure $\mu$ coincides with $\mu_{\eta}$. Proof. See Hutchinson [8] or Falconer [3].

Now we define a post critically finite self-similar set, which is the main object of our study.

Definition 1.12. Let $K$ be a self-similar set. Then $K$ is said to be post critically finite, or p.c.f. for short, if the post critical set $P$ is finite.

If $K$ is p.c.f., it follows immediately by the above definition that $C, P^{(m)}, B_{w}$, and $V_{m}$ are all finite sets and $V_{*}$ is countably infinite. Further, by Lemma 1.7, $K_{w} \cap K_{v}=B_{w} \cap B_{v}$ is finite. So a p.c.f. self-similar set may be called a finitely ramified fractal in physicists' terminology. Nested fractals defined by Lindstrøm [13] are p.c.f. self-similar sets. The set of all essential fixed points in his terminology equals $\pi$ (a post critical set). Lindstrøm's nesting axiom holds for p.c.f. self-similar sets, but the axiom of symmetry is not satisfied in general.

If $K$ is p.c.f., the self-similar measure $\mu_{\eta}$ becomes simple as follows.

Lemma 1.13. Let $K$ be p.c.f. and let $\eta$ satisfy (1.1). Then for all $w \in W_{*}$,

$$
\mu_{\eta}\left(K_{w}\right)=\eta_{w_{1}} \eta_{w_{2}} \cdots \eta_{w_{n}} \text {. }
$$

where $n=|w|$.

Proof. It is obvious from the fact that $\pi^{-1}\left(K_{w}\right)=w \Sigma \cup$ a finite set.

Further discussion on p.c.f. self-similar sets is given in Appendix A. We show, in short, that a p.c.f. self-similar set is determined by

$$
\mathscr{A}=\left(S, V_{1}, V_{0},\left\{\left.F_{s}\right|_{0}\right\}_{s \in S}\right),
$$

which will be called the ancestor of p.c.f. self-similar set.

We will give some examples of p.c.f. self-similar sets in $\S 8$.

\section{QUASI-HARMONIC STRUCTURE}

In this section, we first give the concept of a quasi-harmonic structure which generates difference operators $H_{m}$ on $V_{m}$ and then introduce the notion of the 
harmonic function and quasi-harmonic function as the kernel of the difference operators. Throughout the rest of this paper we fix a p.c.f. self-similar structure $\mathscr{L}=\left(K, S,\left\{F_{s}\right\}_{s \in S}\right)$ and assume that $K$ is connected.

Notations. (1) $l(V)=\{f \mid f: V \rightarrow \mathbb{R}\}$. We use $(f)_{p}$ or $f_{p}$ to denote the value of $f \in l(V)$ at $p \in V$. For $p \in V, \mathbf{e}_{p} \in l(V)$ is defined by

$$
\mathbf{e}_{p}^{V}(q)= \begin{cases}1 & \text { if } q=p \\ 0 & \text { otherwise. }\end{cases}
$$

When no confusion may occur, we write $\mathbf{e}_{p}$ instead of $\mathbf{e}_{p}^{V}$.

In the following, $U$ and $V$ are finite sets.

(2) $L(U, V)=\{A \mid A: l(U) \rightarrow l(V)$ and $A$ is linear $\}$. In particular, $L(V)=$ $L(V, V)$. We use $(A)_{p q}$ or $A_{p q}$ to denote $\left(A \mathbf{e}_{p}\right)_{q}$ for $p \in V$ and $q \in U$. Note that $\sum_{q \in U} A_{p q} f_{q}=(A f)_{p}$. For $A \in L(U, V),{ }^{t} A \in L(V, U)$ is the transpose of $A$.

(3) If $V \cap U=\varnothing$, then we use $U+V$ to denote the disjoint union of $U$ and $V$. Note that $l(U+V)=l(U) \oplus l(V)$, we write

$$
f=\left(\begin{array}{l}
(f)_{U} \\
(f)_{V}
\end{array}\right) \text { for } f \in l(U+V),
$$

where $(f)_{U}=\left.f\right|_{U}$ and $(f)_{V}=\left.f\right|_{V}$.

(4) For $D \in L(V)$ with ${ }^{t} D=D$, we define an equivalence relation $\underset{D}{\sim}$ on $V$ by

(i) $p \underset{D}{\sim} p$ for all $p \in V$.

(ii) For $p \neq q, p \underset{D}{\sim} q$ if and only if there exists $\left\{p_{i}\right\}_{i=1}^{m} \subset V$ with $p_{1}=p$, $p_{m}=q$, and $D_{p_{i} p_{i+1}} \neq 0$ for $i=1,2, \ldots, m-1$.

An equivalence class is called a $D$-irreducible part. Also we say that $D$ is irreducible if and only if $D$ has one irreducible part $V$.

Now we define a quasi-harmonic structure.

Definition 2.1. A pair $(D, r) \in L\left(V_{0}\right) \times l(S)$ is called a quasi-harmonic structure, or q.h.s. for short, on $K$ if and only if

(1) $r_{s}>0$ for each $s \in S$,

(2) ${ }^{t} D=D$,

(3) $D$ is irreducible,

(4) $D_{p p}<0$ and $\sum_{p \in V_{0}} D_{p q}=0$ for each $p \in V_{0}$,

(5) $D_{p q} \geq 0$ if $p \neq q$.

Further, for a finite set $V$, we define $\mathscr{H}(V) \subset L(V)$ by $\mathscr{H}(V)=\{D \mid D$ satisfies (2) $\sim(5)$ where $V_{0}$ is replaced by $V$ \}.

In this section we treat a fixed q.h.s. $(D, r)$.

The bijection $F_{w}: V_{0} \rightarrow B_{w}$ induces a natural isomorphism $\left(F_{w}\right)_{*}: l\left(V_{0}\right) \simeq$ $l\left(B_{w}\right)$. By this identification, we think of $D$ as an element of $L\left(B_{w}\right)$. Then we obtain a difference operator $H_{m}$ on $V_{m}$ form the q.h.s. $(D, r)$ as follows.

Definition 2.2. A difference operator $H_{m} \in L\left(V_{m}\right)$ is defined by

$$
H_{m}=\sum_{w \in W_{m}} r_{w}^{-1} \cdot{ }^{t} R_{w} D R_{w}
$$


where $R_{w} \in L\left(V_{m}, B_{w}\right)$ is the restriction defined by $\left(R_{w} f\right)=\left.f\right|_{B_{w}}$ and $r_{w}=$ $r_{w_{1}} r_{w_{2}} \cdots r_{w_{n}}$. Furthermore, we write, for $m \geq 0$ and $p \in V_{m}, H_{m, p} f=$ $\left(H_{m} f\right)_{p}$, and $H_{p}^{*} f=H_{i(p), p} f$, where $i(p)=\min \left\{n \mid p \in V_{n}\right\}$.

Remark. This definition of difference operators is a natural extension of those on Sierpinski gasket given in [10] to p.c.f. self-similar sets. In fact, if we let

$$
D=\left(\begin{array}{ccc}
-2 & 1 & 1 \\
1 & -2 & 1 \\
1 & 1 & -2
\end{array}\right) \text { and } r=(1,1,1) \text {, }
$$

then

$$
H_{m, p} f=\sum_{q \in V_{m, p}}(f(q)-f(p)),
$$

where $V_{m, p}=\left\{q \mid\left(H_{m}\right)_{p q} \neq 0\right\}$. This coincides with the definition of $H_{m, p} f$ given in [10]. Further details are given in $\S 8$.

Lemma 2.3. For $m \geq 0, H_{m} \in \mathscr{H}\left(V_{m}\right)$.

Proof. (2), (4), and (5) of Definition 2.1 can be verified immediately from the definition of $H_{m}$. We may show that $H_{m}$ is irreducible. Since $D$ is irreducible on $V_{0}, D R_{w}$ is irreducible on $B_{w}$. This implies that $p \underset{H_{m}}{\sim} q$ for $p$ and $q \in B_{w}$ with $w \in W_{m}$. Now let $p \in B_{w}$ and $q \in B_{v}$ with $v, w \in W_{m}$. Then by the fact that $K$ is connected, there exists $\left\{w_{i}\right\}_{i=1}^{k} \subset W_{m}$ with $w_{1}=w, w_{k}=v$, and $K_{w_{i}} \cap K_{w_{i+1}}=B_{w_{i}} \cap B_{w_{i+1}} \neq \varnothing$ for $i=1,2, \ldots, k-1$. Hence, choosing $p_{i} \in B_{w_{i}} \cap B_{w_{i+1}}$, we can chain $p$ and $q$ by $p \underset{H_{m}}{\sim} p_{1}, p_{i} \underset{H_{m}}{\sim} p_{i+1}$, and $p_{k} \underset{H_{m}}{\sim} q$. This completes the proof of the lemma.

The above lemma shows that if $H_{m, p} f=0$, then $f(p)$ is a kind of average of the $f(q)$ 's where $\left(H_{m}\right)_{p q} \neq 0$. This observation motivates the following definition of a harmonic function.

Definition 2.4. A continuous function $f$ on $K$ is said to be harmonic if and only if $\left(H_{m} f\right)_{V_{m}^{\circ}}=0$ for all $m \geq 1$.

It is unfortunate that the difference equations defining a harmonic function are overdetermined in general, and we may scarcely expect that there exist nontrivial harmonic functions without some further assumptions on the q.h.s. $(D, r)$. This problem is treated in $\S 4$. For a while, we investigate functions satisfying some necessary conditions for being harmonic.

Definition 2.5. A continuous function $f$ on $K$ is said to be quasi-harmonic, or q.h. for short, if and only if $H_{p}^{*} f=0$ for all $p \in V_{*}^{\circ}$.

Theorem 2.6. Suppose that

$$
\#\left(B_{s} \cap V_{0}\right) \leq 1 \text { for each } s \in S,
$$

where \# $(A)$ is the number of elements in $A$. Then for each $\rho \in l\left(V_{0}\right)$, there exists a unique quasi-harmonic function $f$ with $\left.f\right|_{V_{0}}=\rho$.

Note that (1) is a condition not on q.h.s. but on the self-similar structure. The proof of Theorem 2.6 is given in Appendix B. We observe some heuristic arguments below and will show a part of them later on. 
First we decompose $H_{m}$ into

$$
H_{m} f=\left(\begin{array}{cc}
T_{m} & { }^{t} J_{m} \\
J_{m} & X_{m}
\end{array}\right)\left(\begin{array}{l}
(f)_{V_{0}} \\
(f)_{V_{m}^{\circ}}
\end{array}\right),
$$

where $T_{m} \in L\left(V_{0}\right), J_{m} \in L\left(V_{0}, V_{m}^{\circ}\right)$, and $X_{m} \in L\left(V_{m}^{\circ}\right)$. In particular, we write $T=T_{1}, J=J_{1}$, and $X=X_{1}$.

Now let $f$ be q.h., write $\left.f\right|_{V_{0}}=f_{0}$ and $\left.f\right|_{V_{1}^{\circ}}=f_{1}$, and consider a procedure to express $f_{1}$ by $f_{0}$. Obviously, for $p \in V_{1}^{\circ}, i(p)=1$, and $H_{p}^{*} f=\left(J f_{0}+\right.$ $\left.X f_{1}\right)_{p}$. Hence, if $X$ is invertible, we can obtain that $f_{1}=-X^{-1} J f_{0}$. So we have

$$
(f)_{V_{1}}=\left(\begin{array}{c}
I \\
-X^{-1} J
\end{array}\right)(f)_{V_{0}},
$$

where $I$ is the identity in $L\left(V_{0}\right)$. Furthermore, noting that $V_{0}=B_{\varnothing}$, we have, for each $s \in S$,

$$
(f)_{B_{s}}=A_{s}(f)_{B_{\varnothing}},
$$

where $A_{s}=R_{s}\left(\begin{array}{c}I \\ -X^{-1} J\end{array}\right)$.

Next, letting $S_{w}=F_{w}\left(V_{1}^{\circ}\right)$, then the above procedure will turn out to be effective in getting $\left.f\right|_{S_{w}}$ from $\left.f\right|_{B_{w}}$. Here we introduce notation and observe some facts about $B_{w}$ and $S_{w}$ before stating the procedure.

(1) We denote $\left.f\right|_{B_{w}}$ by $(f)_{w}^{b}$ or $f_{w}^{b}$ and also denote $\left.f\right|_{S_{w}}$ by $(f)_{w}^{s}$ or $f_{w}^{s}$. When no confusion can arise, we use $f^{b}$ and $f^{s}$ instead of $f_{w}^{b}$ and $f_{w}^{s}$.

(2) Using the bijections $F_{w}: V_{0} \rightarrow B_{w}$ and $\left.F_{w}\right|_{V_{1}^{\circ}}: V_{1}^{\circ} \rightarrow S_{w}$, we always identity

$$
\begin{aligned}
L\left(B_{w}\right) & \simeq L\left(V_{0}\right), \quad L\left(B_{w}, S_{w}\right) \simeq L\left(V_{0}, V_{1}^{\circ}\right), \\
L\left(S_{w}, B_{w}\right) & \simeq L\left(V_{1}^{\circ}, V_{0}\right), \quad L\left(S_{w}\right) \simeq L\left(V_{1}^{\circ}\right) .
\end{aligned}
$$

(3) $D_{w}^{*} \in L\left(B_{w}+S_{w}, B_{w}\right)$ and $H_{w}^{*} \in L\left(B_{w}+S_{w}, S_{w}\right)$ is defined by

$$
\left(\begin{array}{l}
D_{w}^{*} \\
H_{w}^{*}
\end{array}\right)=r_{w}^{-1}\left(\begin{array}{cc}
T & t \\
J & X
\end{array}\right)\left(\begin{array}{l}
f_{w}^{b} \\
f_{w}^{s}
\end{array}\right) .
$$

Then, we can easily see that, for $p \in S_{w}$,

$$
i(p)=|w|+1 \text { and } H_{p}^{*} f=\left(H_{u}^{*} f\right)_{p} .
$$

Now applying the above procedure, we obtain that $f^{s}=-X^{-1} J f^{b}$ for a quasi-harmonic function $f$. Further (2.2) becomes

$$
f_{w s}^{b}=A_{s} f_{w}^{b},
$$

for all $w \in W_{*}$ and $s \in S$. Hence we can see that

$$
f_{w}^{b}=A_{w}(f)_{V_{0}},
$$

where $A_{w}=A_{w_{n}} A_{w_{n-1}} \cdots A_{w_{1}}$.

From the preceding discussions, we next show some results about $X_{m}$.

Lemma 2.7. For each $m \geq 1$,

(1) $X_{m}$ is invertible and $X_{m}^{-1} \leq 0$, where $A \leq 0$ means $A_{p q} \leq 0$ for all $p$ and $q$.

(2) Let $g_{p q}^{m}=-\left(X_{m}^{-1}\right)_{p q}$ for $p$ and $q \in V_{m}^{\circ}$. Then $g_{p p}^{m} \geq g_{p q}^{m}$ for each $q \in V_{m}^{\circ}$.

We will prove Lemma 2.7 by using the following fact. 
Lemma 2.7.1. Let $V$ be a finite set and let $X \in L(V)$. Suppose that

(I) ${ }^{t} X=X$ and $X$ is irreducible,

(II) $X_{p p}<0$ and $\sum_{q \in V} X_{p q} \leq 0$ for any $p \in V$,

(III) $X_{p q} \geq 0$ if $p \neq q$,

(IV) $\sum_{q \in V} X_{p q}<0$ for some $p \in V$.

Then we have

(1) $X$ is invertible and $X^{-1} \leq 0$.

(2) Let $g_{p q}=\left(-X^{-1}\right)_{p q}$ for $p$ and $q \in V$. Then, $g_{p p} \geq g_{p q}$.

Proof. Let

$$
x_{p q}= \begin{cases}X_{p q} & \text { if } p \neq q, \\ -X_{p q} & \text { if } p=q,\end{cases}
$$

and let $y_{p}=\sum_{q \neq p} x_{p q} / x_{p p}$. Then (II) implies

$$
0<y_{p} \leq 1 \text { for all } p \in V,
$$

and (IV) becomes

$(\text { IV })^{\prime}$

$$
0<y_{p}<1 \text { for some } p \in V .
$$

To prove (1), it suffices to show that

$$
\text { if } X f \geq 0, \text { then } f \leq 0 \text {, }
$$

because (2.5) implies that $X f=0$ if and only if $f=0$ and $X^{-1} f \leq 0$ for any $f \geq 0$. Now assuming that $X f \geq 0$ and $\max _{q \in V} f_{q}=M>0$. Then choosing $p \in V$ with $f_{p}=M$, we have

$$
y_{p} f_{p} \geq \sum_{q \neq p}\left(x_{p q} / x_{p p}\right) f_{q} \geq f_{p} .
$$

Note that from $f_{p}=M>0$ and $f_{p} \geq f_{q}$, we can see that

$$
y_{p}=1 \text { and } f_{p}=f_{q} \text { if } x_{p q} \neq 0 .
$$

Since $X$ is irreducible, the above discussion implies inductively that $y_{q}=1$ for all $q \in V$. This contradicts (IV)' . Thus we have shown (2.5). To prove (2), fix $p \in V$, let $d_{q}=g_{p q}$ for each $q \in V$ and assume that $M=\max _{q \in V} d_{q}>d_{p}$. Then choosing $r \in V$ with $d_{r}=M$, as $g_{p q}=\left(-X^{-1}\right)_{p q}$, we have

$$
\sum_{q \neq r} x_{r q} d_{q}-x_{r r} d_{r}=0
$$

and hence

$$
d_{r} \leq \sum_{q \neq r}\left(x_{r q} / x_{r r}\right) d_{q} \leq y_{r} d_{r} .
$$

Since $d_{r}=M \geq d_{q} \geq 0$ for $q \neq r$, we can conclude that

$$
y_{r}=1, \quad d_{q}=M \text { if } x_{r q} \neq 0, \quad \text { and } \quad x_{r p}=0 .
$$

By the fact that $X$ is irreducible, the above discussion implies inductively that $x_{q p}=0$ for all $q \neq p$. This contradicts to the fact that $X$ is irreducible. Therefore we can conclude Lemma 2.7.1. 
Proof of Lemma 2.7. $X_{m}$ can be decomposed into irreducible parts, that is,

$$
V_{m}^{\circ}=V_{m}^{(1)}+V_{m}^{(2)}+\cdots+V_{m}^{\left(i_{m}\right)} \text {, }
$$

and

$$
X_{m}=X_{m}^{(1)} \oplus X_{m}^{(2)} \oplus \cdots \oplus X_{m}^{\left(i_{m}\right)},
$$

where $V_{m}^{(i)}$ is an $X_{m}$-irreducible part and $X_{m}^{(i)} \in L\left(V_{m}^{(i)}\right)$. Then it suffices to show that each $X_{m}^{(i)}$ satisfies the assumptions of Lemma 2.7.1. Using Lemma 2.3 and (2.1), we can easily verify (I), (II), and (III). Since $H_{m}$ is irreducible, for each $i$, we can choose $p_{*} \in V_{m}^{(i)}$ and $q_{*} \in V_{0}$ with $\left(H_{m}\right)_{p_{*} q_{*}}>0$.

Noting that $\sum_{q \in V_{m}}\left(H_{m}\right)_{p_{*} q}=0$, we have $\sum_{q \in V_{m}^{\circ}}\left(X_{m}\right)_{p_{*} q} \leq-\left(H_{m}\right)_{p_{*} q_{*}}<0$. Therefore we can conclude that (IV) holds for each $X_{m}^{(i)}$.

The last lemma will play an important role in $\S 5$. We next give a result on $A_{s}$.

Lemma 2.8. For each $s \in S, A_{s}$ is a stochastic matrix, that is, $A_{s} \geq 0$ and $A_{s} \mathbf{e}=\mathbf{e}$, where $\mathbf{e}=\sum_{p \in V_{0}} \mathbf{e}_{p}$.

Proof. As $X^{-1} \leq 0$ and $J \geq 0$, we have $A_{s} \geq 0$. Next for the q.h. function $f \equiv 1,(f)_{s}^{b}=(f)_{V_{0}}=\mathbf{e}$. Hence (2.2) implies $A_{s}=\mathbf{e}$.

As an immediate consequence of Lemma 2.8, we have the "maximal principle" for quasi-harmonic functions as follows.

Theorem 2.9. Let $f$ be quasi-harmonic. Then, for any $x$ in $K$, we have

$$
\min _{p \in V_{0}} f(p) \leq f(x) \leq \max _{p \in V_{0}} f(p) .
$$

At the end of this section, we introduce the notion of piecewise quasiharmonic functions.

Definition 2.10. A continuous function $f$ on $K$ is said to be $m$-quasi-harmonic, or $m$-q.h. for short, if and only if $f \circ F_{w}$ is quasi-harmonic for all $w \in W_{m}$.

\section{QUASI-HARMONIC EXPANSION}

This section is devoted to the expansion of a function by a system of piecewise quasi-harmonic functions. This is called a quasi-harmonic expansion, or q.h. expansion for short. We always fix a p.c.f. self-similar structure $\left(K, S,\left\{F_{s}\right\}_{s \in S}\right)$ and a q.h. structure $(D, r)$ on $K$. Further we will need sufficiently many piecewise quasi-harmonic functions for the q.h. expansion. So, through this section, we assume that

Assumption (2). There exists a unique q.h. function $f$ with $\left.f\right|_{V_{0}}=\rho$ for any $\rho \in l\left(V_{0}\right)$. have

We have shown in Theorem 2.6 that (1) implies (2). Now assuming (2), we

Theorem 3.1. For each $\rho \in l\left(V_{m}\right)$, there exists a unique m-q.h. $f$ with $\left.f\right|_{V_{m}}=$ $\rho$. Especially, for each $p \in V_{m}$, we denote the unique m-q.h. $f$ with $\left.f\right|_{V_{m}}=\mathbf{e}_{p}$ by $\psi_{p}^{m}$. Then for any $\rho \in l\left(V_{m}\right)$, the unique m-q.h. $f$ with $\left.f\right|_{V_{m}}=\rho$ is given by $f=\sum_{p \in V_{m}} \rho_{p} \psi_{p}^{m}$. 
We will use $\left\{\psi_{p}\right\}_{p \in V_{*}}$ as the basis of the q.h. expansion, where $\psi_{p}=\psi_{p}^{i(p)}$. Using Theorem 2.9, we can obtain another important property of $m$-q.h. functions, which is called the maximal principle.

Theorem 3.2. Let $f$ be m-q.h. Then, for each $w \in W_{*}$ with $|w| \geq m$ and for any $x \in K_{w}$,

$$
\min _{q \in B_{w}} f(q) \leq f(x) \leq \max _{q \in B_{w}} f(q) .
$$

Definition 3.3. Let $f \in l\left(V_{*}\right)$. Then $\alpha \in l\left(V_{*}\right)$ is called a quasi-harmonic expansion of $f$ if, for all $q \in V_{*}$,

$$
\sum_{p \in V_{m}} \alpha_{p} \psi_{p}(q) \rightarrow f(q) \quad \text { as } m \rightarrow \infty
$$

When no confusion may occur, we denote $\left.\psi_{p}\right|_{V_{*}}$ by $\psi_{p}$. Also, we denote $H_{w}^{*}\left(\left.f\right|_{B_{w}+S_{w}}\right)$ by $H_{w}^{*} f$.

In the following, we show that every $f \in l\left(V_{*}\right)$ has a unique q.h. expansion.

Lemma 3.4. Let $f \in l\left(V_{*}\right)$. If $\alpha \in l\left(V_{*}\right)$ is a q.h. expansion of $f$, then

$$
\begin{cases}\alpha_{p}=f(p) & \text { for each } p \in V_{0}, \\ \alpha_{w}^{s}=r_{w} X^{-1} H_{w}^{*} f & \text { for each } w \in W_{*} .\end{cases}
$$

Lemma 3.4.1. For $w \in W_{m}$ and $p \in V_{*}$,

$$
H_{w}^{*} \psi_{p}= \begin{cases}r_{w}^{-1} X \mathbf{e}_{p} & \text { if } p \in S_{w}, \\ 0 & \text { otherwise. }\end{cases}
$$

Proof. First if $p \in S_{w}$, then

$$
H_{w}^{*} \psi_{p}=r_{w}^{-1}\left(J\left(\psi_{p}\right)^{b}+X\left(\psi_{p}\right)^{s}\right)=r_{w}^{-1} X \mathbf{e}_{p} .
$$

Next, if $p$ does not belong to $S_{w}$ and $i(p) \geq|w|+1$, then $\left.\psi_{p}\right|_{B_{w}+S_{w}}=0$. Therefore $H_{w}^{*} \psi_{p}=0$. Finally, if $i(p)<m$, then $\psi_{p}$ is $i(p)$-q.h. and therefore $H_{w}^{*} \psi_{p}=0$.

Proof of Lemma 3.4. Let $f_{m}=\sum_{p \in V_{m}} \alpha_{p} \psi_{p}$, then for all $w \in W_{*}$,

$$
\lim _{m \rightarrow \infty} H_{w}^{*} f_{m}=H_{w}^{*} f
$$

and for each $p \in V_{0}, f_{m}(p)=\alpha_{p}=f(p)$.

On the other hand, using Lemma 3.4.1, we have, for $m \geq|w|+1$,

$$
H_{w}^{*} f_{m}=\sum_{p \in S_{w}} \alpha_{p} r_{w}^{-1} X \mathbf{e}_{p}=r_{w}^{-1} X \sum_{p \in S_{w}} \alpha_{p} \mathbf{e}_{p}=r_{w}^{-1} X \alpha_{w}^{s} .
$$

Letting $m \rightarrow \infty$, we obtain $\alpha_{w}^{s}=r_{w} X^{-1} H_{w}^{*} f$ as required.

The above lemma says that each $f \in l\left(V_{*}\right)$ has at most one q.h. expansion given by (3.1). So we define $\alpha(f) \in l\left(V_{*}\right)$ by

$$
\begin{cases}(\alpha(f))_{p}=f(p) & \text { for } p \in V_{0}, \\ (\alpha(f))_{w}^{s}=r_{w} X^{-1} X_{w}^{*} f & \text { for } w \in W_{*} .\end{cases}
$$

We denote $(\alpha(f))_{w}^{s}$ by $\alpha_{w}(f)$. We also define, for all $m \geq 0, P_{m} f=$ $\sum_{p \in V_{m}}(\alpha(f))_{p} \psi_{p}$. 
Lemma 3.5. Let $f \in l\left(V_{*}\right)$, then $P_{m} f=\sum_{p \in V_{m}} f(p) \psi_{p}^{m}$.

Proof. Since $P_{m} f$ is $m$-q.h., by Theorem 3.1, we may show by induction on $m$ that

$$
\left.f\right|_{V_{m}}=\left.\left(P_{m} f\right)\right|_{V_{m}} \text {. }
$$

$(3.2)_{0}$ is obvious by definition. Now suppose $(3.2)_{m}$. Note that

$$
P_{m+1} f=P_{m} f+\sum_{p \in V_{m+1}-V_{m}}(\alpha(f))_{p} \psi_{p} .
$$

Hence, if $p \in V_{m}$, then $\left(P_{m+1} f\right)_{p}=\left(P_{m} f\right)_{p}=f(p)$. Next for each $w \in W_{m}$,

$$
\left(P_{m} f\right)_{w}^{s}=-X^{-1} J\left(P_{m} f\right)_{w}^{b}=-X^{-1} J f_{w}^{b} .
$$

This together with (3.3) shows that

$$
\left(P_{m+1} f\right)_{w}^{s}=\left(P_{m} f\right)_{w}^{s}+\alpha_{w}(f)=-X^{-1} J f_{w}^{b}+X^{-1}\left(J f_{w}^{b}+X f_{w}^{s}\right)=f_{w}^{s} .
$$

This implies that $\left(P_{m+1} f\right)_{p}=f(p)$ for any $p \in V_{m+1}-V_{m}$.

Combining Lemma 3.4 and Lemma 3.5, we have

Theorem 3.6. Each $f \in l\left(V_{*}\right)$ has the unique quasi-harmonic expansion $\alpha(f)$.

In the rest of this section, we consider q.h. expansion for continuous functions. The set of all continuous functions on $K$ is denoted by $C(K)$, which is equipped with the supremum norm defined by $|f|_{K}=\sup _{x \in K}|f(x)|$.

Since $V_{*}$ is dense in $K$, the restriction map $i: C(K) \rightarrow l\left(V_{*}\right)$ given by $i(f)=\left.f\right|_{V_{*}}$ becomes an inclusion map. Hereafter, $C(K)$ is regarded as a subset of $l\left(V_{*}\right)$ in this manner. We also regard $l\left(V_{m}\right)$ as a subset of $l\left(V_{*}\right)$ by the natural map $i_{m}$ defined by, for $\rho \in V_{m}, i_{m}(\rho)=\sum_{p \in V_{m}} \rho_{p} \psi_{p}^{m}$. Then, $P_{m}: l\left(V_{*}\right) \rightarrow l\left(V_{m}\right)$ defined above equals to the projection map for the inclusion $i_{m}$.

Noting that $l\left(V_{m}\right) \subset C(K)$, we have

Theorem 3.7. Let $f \in l\left(V_{*}\right)$. Then $f \in C(K)$ if and only if $P_{m} f$ is uniformly convergent on $K$ as $m \rightarrow \infty$. And if $f \in C(K)$, then $P_{m} f$ converges to $f$ uniformly on $K$.

Proof. If $P_{m} f$ is uniformly convergent on $K$ as $m \rightarrow \infty$, then the limit, say, $\tilde{f}$ is continuous on $K$. Therefore from

$$
\left.\tilde{f}\right|_{V_{m}}=\left.\left(P_{m} f\right)\right|_{V_{m}}=\left.f\right|_{V_{m}}
$$

we have $\tilde{f}=f$ and $f \in C(K)$.

Next if $f \in C(K)$, let

$$
\varepsilon_{m}=\max _{w \in W_{m}} \sup _{x, y \in K_{w}}|f(x)-f(y)| .
$$

Then since $f$ is uniformly continuous on $K, \lim _{m \rightarrow \infty} \varepsilon_{m}=0$. On the other hand, using Theorem 3.2, we can obtain, for any $x$,

$$
\left|P_{m} f(x)-f(x)\right| \leq\left|P_{m} f(x)-f(p)\right|+|f(p)-f(x)| \leq 2 \varepsilon_{m},
$$

where $p$ is chosen so that $x \in K_{w}$ and $p \in B_{u}$ for some $w \in W_{m}$. Hence $\left|P_{m} f-f\right|_{K} \leq 2 \varepsilon_{m}$ and so $P_{m} f$ converges to $f$ uniformly on $K$. 


\section{HARMonic STRUCTURE}

In this section, we introduce the concept of harmonic structure, which has all the properties required in the further study of calculus on p.c.f. self-similar sets. For example, under harmonic structure, each quasi-harmonic function will turn out to be harmonic.

Definition 4.1. Let $(D, r)$ be a quasi-harmonic structure. Then $\mathscr{I}_{r}(D) \in L\left(V_{0}\right)$ is defined by

$$
\mathscr{I}_{r}(d)=T-{ }^{t} J X^{-1} J
$$

Harmonic structure will be defined as an eigenvector of the nonlinear transformation $\mathscr{I}_{r}$. When no confusion can arise, we drop the $r$ of $\mathscr{I}_{r}$ and write $\mathscr{I}$.

Lemma 4.2. For each $w \in W_{*}$ and any $f \in l\left(B_{w}+S_{w}\right)$,

$$
r_{w}^{-1} \mathscr{J}(D) f_{w}^{b}=D_{w}^{*} f-{ }^{t} J X^{-1} H_{w}^{*} f \text {. }
$$

Proof. This follows immediately by the definition of $D_{w}^{*}$ and $H_{w}^{*}$ in $\S 2$.

The following result will not be applied in this paper. It implies, however, the notable fact that $\mathscr{I}_{r}: \mathscr{H}\left(V_{0}\right) \rightarrow \mathscr{H}\left(V_{0}\right)$, which may be a starting point of some further study.

Proposition 4.3. For each quasi-harmonic structure $(D, r),\left(\mathscr{F}_{r}(D), r\right)$ is also a quasi-harmonic structure.

For the proof of Proposition 4.3, we decompose $V_{1}^{\circ}$ into $X$-irreducible parts $U_{1}, U_{2}, \ldots, U_{m}$ and we define

$$
\partial U_{i}=\left\{p \mid p \in V_{0} \text { and } J_{p q} \neq 0 \text { for some } q \in U_{i}\right\} \text {. }
$$

Lemma 4.3.1. For $p \in V_{0}$, let $\psi_{p} \in l\left(V_{1}\right)$ be the quasi-harmonic function with $\left.\psi_{p}\right|_{V_{0}}=\mathbf{e}_{p}$. Then, for $q \in V_{1}^{\circ}, \psi_{p}(q)>0$ if, and only if $q \in U_{i}$ and $p \in \partial U_{i}$ for some $i=1,2, \ldots, m$.

Proof. First if $q \in U_{i}$ and $p \in\left(\partial U_{i}\right)^{c}$, then $\left.\psi_{p}\right|_{\partial U_{i}} \equiv 0$ and so $\psi_{p}(q)=0$. Hence if $\psi_{p}(q)>0$ then $q \in U_{i}$ and $p \in \partial U_{i}$ for some $i=1,2, \ldots, m$. Conversely, if $q \in U_{i}$ and $\psi_{p}(q)=0$, then $\psi_{p}(q)=\min _{r \in V_{1}} \psi_{p}(r)$. As $H_{1, q} \psi_{p}=0, \psi_{p}(r)=0$ for all $r$ with $\left(H_{1}\right)_{q r} \neq 0$. Inductively, we can show that $\left.\psi_{p}\right|_{U_{i} \cup \partial U_{i}} \equiv 0$. Therefore $p \in\left(\partial U_{i}\right)^{c}$.

Using Lemma 4.2 , we have $\mathscr{S}(D) \mathbf{e}_{p}=D_{w}^{*} \psi_{p}$, and hence, for all $p$ and $r \in V_{0}$,

$$
\mathscr{I}(D)_{r p}=h_{r p}+\sum_{q \in V_{1}^{\circ}} h_{r q} \psi_{p}(q)
$$

where $h_{a b}=\left(H_{1}\right)_{a b}$.

Lemma 4.3.2. For $p$ and $r \in V_{0}$, if $p$ and $r \in \partial U_{i}$ for some $i=1,2, \ldots, m$, then $\mathscr{I}(D)_{r p}>0$.

Proof. There exists $q \in U_{i}$ with $h_{r q}>0$. And by Lemma 4.3.1, $\psi_{p}(q)>0$. Hence by $(4.1), \mathscr{I}(D)_{r p}>0$.

Proof of Lemma 4.3. We may show that $\mathscr{I}_{r}(D) \in \mathscr{H}\left(V_{0}\right)$. Noting that $-{ }^{t} J X^{-1} J$ $\geq 0$ and $\mathscr{I}(D) \mathbf{e}=0$, where $\mathbf{e}=\sum_{p \in V_{0}} \mathbf{e}_{p}$, we can easily verify that (2), (4), 
and (5) of Definition 2.1 hold. Therefore we may show that $\mathscr{I}(D)$ is irreducible. Then, for $p$ and $q \in V_{0}$, as $H_{1}$ is irreducible, there is a sequence $\left\{q_{i}\right\}_{i=1}^{n}$ such that $q_{1}=p, q_{n}=q$, and $h_{q_{i} q_{i+1}}>0$ for $i=1,2, \ldots, n-1$. Let $\left\{q_{i}\right\}_{i=1}^{n} \cap V_{0}=\left\{p_{i}\right\}_{i=1}^{k}$ where $p_{i}=q_{i_{k}}$ with $1=i_{1}<i_{2}<\cdots<i_{k}=n$.

Now if $i_{j+1}=i_{j}+1$, then $h_{p_{j} p_{j+1}}>0$ and hence by (4.1) we have $p_{j} \tilde{\mathscr{I}(D)}$ $p_{j+1}$. If $i_{j+1}>i_{j}+1$, then $p_{j}$ and $p_{j+1} \in \partial U_{i}$ for some $i$ and by Lemma 4.3.2, we have $p_{j} \underset{\mathcal{F}(D)}{\sim} p_{j+1}$. Therefore we have $p_{\mathcal{I}(\widetilde{D})}^{\sim} q$. This completes the proof.

Definition 4.4. A quasi-harmonic structure $(D, r)$ is said to be a harmonic structure if there exists $\lambda>0$ such that

$$
\mathscr{F}_{r}(d)=\lambda^{-1} D
$$

This $\lambda$ is called the characteristic exponent of the harmonic structure $(D, r)$ and is denoted by $\lambda=\lambda(D, r)$. Further, a harmonic structure $(D, r)$ is said to be regular if $r_{s}<\lambda(D, r)$ for each $s \in S$.

Remark. One may ask whether there is any harmonic structure on a given p.c.f. self-similar set. In general, we are not ready to answer this question. In the case of nested fractal, Lindstrøm has treated essentially the same problem in [13]. In our words, he has shown that there exists a regular symmetric harmonic structure on every nested fractal. K. Hattori et al. [7] has also treated a similar problem by another approach. From their discussion, we can deduce that there exists a p.c.f. self-similar set where $\mathscr{J}_{r}$ has no eigenvector with positive eigenvalue for some $r$.

Examples of harmonic structures are given in $\S 8$. We now give some remarkable facts derived from the definition of harmonic structure.

Lemma 4.5. Let $(D, r)$ be a harmonic structure with $\lambda=\lambda(D, r)$. If $f$ is $m$-q.h., then, for each $p \in V_{m}$ and $k \geq 0, H_{m+k, p} f=\lambda^{-k} H_{m, p} f$.

Proof. Using induction on $k$, we need to show only the case when $k=1$. For $w \in W_{*}$ with $p \in B_{w}$, by Lemma 4.2, we have

$$
r_{w}^{-1}\left(D f_{w}^{b}\right)_{p}=\lambda\left(D_{w}^{*} f-{ }^{t} J X^{-1} H_{w}^{*} f\right)_{p}
$$

As $f$ is $m$-q.h., we obtain $r_{w}^{-1}\left(D f_{w}^{b}\right)_{p}=\lambda\left(D_{w}^{*} f\right)_{p}$. Here, summing the above equality for all $w \in W_{*}$ with $p \in B_{w}$, we have $H_{m, p} f=\lambda H_{m+1, p} f$ as required.

Lemma 4.6. If $(D, r)$ is a harmonic structure, then every quasi-harmonic function is harmonic.

Proof. If $f$ is q.h., then Lemma 4.5 implies that for all $p \in V_{*}$ and all $m \geq$ $i(p)$,

$$
H_{m, p} f=\lambda^{-(m-i(p))} H_{p}^{*} f=0 .
$$

Theorem 4.7. Let $(D, r)$ be a harmonic structure. Then for any $\rho \in l\left(V_{0}\right)$, there exists a unique harmonic function $f$ with $\left.f\right|_{V_{0}}=\rho$.

Proof. If the assumption (1) in Theorem 2.6 is satisfied, then combining Theorem 2.6 and Lemma 4.6, we can deduce the above result. In the general case, we let $\mathscr{L}_{m}=\left(K, S_{m},\left\{F_{w}\right\}_{w \in S_{m}}\right)$, where $\mathscr{L}=\left(K, S,\left\{F_{s}\right\}_{s \in S}\right)$ is the original selfsimilar structure and $S_{m}=W_{m}(s)$. Also, for the original harmonic structure $(D, r)$, we let $r^{(m)}=\left(r_{w}\right)_{w \in S_{m}}$. 
Then, $\mathscr{L}_{m}$ is a self-similar structure and $\left(D, r^{(m)}\right)$ is a harmonic structure on $\mathscr{L}_{m}$. Now we can easily see that $\mathscr{L}_{m}$ satisfies (1) for sufficiently large $m$. And so, by the above discussion, there exists a unique $\mathscr{L}_{m}$-harmonic function $f$ with $\left.f\right|_{V_{0}}=\rho$ for each $\rho \in l\left(V_{0}\right)$, where $\mathscr{L}_{m}$-harmonic means harmonic with respect to $\mathscr{L}_{m}$ and $\left(D, r^{(m)}\right)$. We may prove that $\mathscr{L}_{m}$-harmonic function is $\mathscr{L}$-harmonic. Noting that

$$
V_{0}\left(\mathscr{L}_{m}\right)=V_{0}(\mathscr{L}), \quad V_{n}\left(\mathscr{L}_{m}\right)=V_{n m}(\mathscr{L}), \quad \text { and } \quad H_{n}\left(\mathscr{L}_{m}\right)=H_{n m}(\mathscr{L}),
$$

Lemma 4.6 implies that if $f$ is $\mathscr{L}_{m}$-harmonic, for all $n \geq 0$,

$$
\left(H_{n m}(\mathscr{L}) f\right)_{V_{n m}^{\circ}}=0 .
$$

On the other hand, using Lemma 4.2, we can show that if

$$
\left(H_{k}(\mathscr{L}) f\right)_{V_{k}^{\circ}}=0 \text { for some } k \geq 0 \text {, }
$$

then

$$
\left(H_{j}(\mathscr{L}) f\right)_{V_{j}^{\circ}}=0 \text { for all } j \leq k .
$$

This together with (4.2) shows that $f$ is $\mathscr{L}$-harmonic.

By the last theorem, we can use the theory of quasi-harmonic expansion in $\S 3$ under a harmonic structure. Hereafter, if $(D, r)$ is a harmonic structure, we drop "quasi-" of quasi-harmonic function, $m$-quasi-harmonic function, quasiharmonic expansion and so forth.

The following two results will play an important role in $\S 5$ and $\S 6$.

Lemma 4.8. Let $(D, r)$ be a harmonic structure with $\lambda=\lambda(D, r)$. Then, for all $p \in V_{m}$ and $q \in V_{n}$,

$$
\lambda^{m} H_{m, p} \psi_{q}^{n}=\lambda^{n} H_{n, q} \psi_{p}^{m} .
$$

Proof. First if $m=n$, then $H_{m, p} \psi_{q}^{n}=\left(H_{m} \mathbf{e}_{q}\right)_{p}=\left(H_{m}\right)_{p q}$. Since ${ }^{t} H_{m}=H_{m}$, we have $H_{m, p} \psi_{q}^{n}=H_{n, q} \psi_{p}^{m}$.

Next, if $m \neq n$, suppose $m>n$, then $\psi_{q}^{n}$ is $n$-harmonic and hence Lemma 4.5 implies that $H_{m, p} \psi_{q}^{n}=\lambda^{-(m-n)} H_{n, p} \psi_{q}^{n}$. Applying the result when $m=n$ and noting that $\left.\psi_{p}^{m}\right|_{V_{n}}=\left.\psi_{p}^{n}\right|_{V_{n}}$,

$$
H_{m, p} \psi_{q}^{n}=\lambda^{-(m-n)} H_{n, q} \psi_{p}^{n}=\lambda^{-(m-n)} H_{n, q} \psi_{p}^{m} .
$$

This completes the proof of Lemma 4.8.

Lemma 4.9. If $(D, r)$ is a harmonic structure with $\lambda=\lambda(D, r)$, then for any $m \geq 1$, we have

$$
T_{m}-{ }^{t} J_{m} X_{m}^{-1} J_{m}=\lambda^{-m} D .
$$

Proof. By Theorem 4.7, for each $\rho \in l\left(V_{0}\right)$, there exists a harmonic function $f$ with $\left.f\right|_{V_{0}}=\rho$. Making use of Lemma 4.5, we have $\left(H_{m} f\right)_{V_{0}}=\lambda^{-m} D \rho$. Noting that $\left.f\right|_{V_{m}^{\circ}}=-X_{m}^{-1} J_{m} \rho$, we obtain

$$
\left(H_{m} f\right)_{V_{0}}=\left(T_{m}-{ }^{t} J_{m} X_{m}^{-1} J_{m}\right) \rho .
$$

Consequently, we can get the required equality.

As is shown in $\S 8$, a harmonic structure is not always regular. We can see, however, the following result. 
Theorem 4.10. If $(D, r)$ is a harmonic structure, then $r_{s}<\lambda(D, r)$ for some $s \in S$.

Theorem 4.10 will be used for the proof of existence of "admissible" measures in the case of nonregular harmonic structure in $\S 5$.

Lemma 4.10.1. Let $\mathscr{L}=\left(K, S,\left\{F_{s}\right\}_{s \in S}\right)$ be a p.c.f. self-similar structure. If $\omega \in \Sigma$ is periodic, that is, $\sigma^{n}(\omega)=\omega$ for some $n$, then $\pi^{-1}(\pi(\omega))=\{\omega\}$.

Proof. For $w \in W_{*}$, we define $w^{k} \in W_{*}$ for $k=1,2, \ldots$ inductively by $w^{1}=w$ and $w^{k+1}=w \cdot w^{k}$. And also, we define $\dot{w} \in \Sigma$ by $\dot{w}=w \cdot w \cdot w \ldots$. Then if $\omega$ is periodic, there exists $w \in W_{*}$ with $\omega=\dot{w}$. Suppose $\tilde{\omega} \in$ $\pi^{-1}(\pi(\omega))$ with $\tilde{\omega} \neq \omega$, then for each $k \geq 1$,

$$
\pi\left(w^{k} \cdot \tilde{\omega}\right)=\pi\left(w^{k} \cdot \omega\right)=\pi(\omega) .
$$

Hence $\pi^{-1}(\pi(\omega))$ contains infinitely many elements. This contradicts to the fact that $\mathscr{L}$ is p.c.f.

Lemma 4.10.2. Let $(D, r)$ be a harmonic structure on $\mathscr{L}=\left(K, S,\left\{F_{s}\right\}_{s \in S}\right)$. If $\mathscr{L}$ satisfies the assumption (1) of Theorem 2.6 then, for any $p \in V_{0},(-\mathscr{I}(D))_{p p}$ $<(-T)_{p p}$.

Proof. Recalling (4.1), we have $(-\mathscr{I}(D))_{p p}=(-T)_{p p}-\sum_{q \in V_{1}^{\circ}} \psi_{p q} h_{q p}$. Using a similar discussion as in the proof of Lemma 4.3, if $h_{p q}>0$, then $\psi_{p q}>0$. Further, if (1) holds, then $\left\{q \mid q \in V_{1}^{\circ}\right.$ and $\left.h_{p q}>0\right\} \neq \varnothing$ and so $\sum_{q \in V_{1}^{\circ}} \psi_{p q} h_{q p}>$ 0 . This completes the proof of Lemma 4.10.2.

Lemma 4.10.3. Let $(D, r)$ be a harmonic structure. If $\dot{w} \in P$ for $w \in W_{*}$, then $r_{w}<\lambda(D, r)^{|w|}$.

Proof. Recalling $H_{1}=\sum_{s \in S} r_{s}^{-1} \cdot{ }^{t} R_{s} D R_{s}$, we have

$$
T_{p p}=\sum_{(q, s) \in Q_{p}} r_{s}^{-1} D_{q q},
$$

where $Q_{p}=\left\{(q, s) \mid q \in V_{0}\right.$ and $\left.F_{s}(q)=p\right\}$. Let $w=w_{1} w_{2} \cdots w_{m}$ with $w_{i} \in S$ and let $p_{i}=\pi\left(\sigma^{i}(\dot{w})\right)$ for $i=1,2, \ldots, m$. Then by Lemma 4.10.1, we obtain, for $i=1,2, \ldots, m, Q_{p_{i}}=\left\{\left(p_{i+1}, w_{i}\right)\right\}$, where $p_{m+1}=p_{1}$. Hence by (4.3),

$$
T_{p_{i} p_{i}}=r_{w_{i}}^{-1} D_{p_{i+1} p_{i+1}} \text {. }
$$

Now suppose the assumption (1) of Theorem 2.6 holds, then using Lemma 4.10.2, we have $T_{p_{i} p_{i}}<\lambda r_{w_{i}}^{-1} T_{p_{i+1} p_{i+1}}$, and so $T_{p_{1} p_{1}}<\lambda^{|w|} r_{w}^{-1} T_{p_{1} p_{1}}$. Consequently $r_{w}<\lambda^{|w|}$.

In the general case, we change the self-similar structure $\mathscr{L}$ by $\mathscr{L}_{m}$ as in the proof of Theorem 4.7. We choose $m$ so that $\mathscr{L}_{m}$ can satisfy (1). Then, by Lemma $4.9, \lambda\left(D, r^{(m)}\right)=\lambda^{m}$. Therefore the above arguments will imply that $r_{w}^{m}<\lambda^{m|w|}$. Hence we have $r_{w}<\lambda^{|w|}$.

Proof of Theorem 4.10. By the definition of $P$, if $\mathscr{L}$ is p.c.f., then $P$ contains a periodic element $\dot{w}$. By Lemma 4.10.3, $r_{w}<\lambda^{|w|}$. And so, $r_{s}<\lambda$ for at least one $s \in S$.

\section{GREEN'S FUNCTION}

In this section, we introduce the Green function $g$ associated with a harmonic structure. And, for some appropriate measure $\mu$, we define the Green 
operator $G_{\mu}$ as an integral operator whose kernel is $g .-G_{\mu}$ will turn out to be the inverse of the Laplace operator in $\S 6$. We always fix a harmonic structure $(D, r)$ with $\lambda=\lambda(D, r)$.

Definition 5.1. (1) For $w \in W_{*}$ and $x \in K$, we define $\psi_{w}(x) \in l\left(S_{w}\right)$ by $\left(\psi_{w}(x)\right)_{p}=\psi_{p}(x)$ for each $p \in S_{w}$.

(2) For $m \geq 1, \Psi_{m}$ and $g_{m}: K \times K \rightarrow \mathbb{R}$ are defined by

$$
\Psi_{m}(x, y)=-\sum_{w \in W_{m-1}} r_{w}{ }^{t} \psi_{w}(x) X^{-1} \psi_{w}(y),
$$

and

$$
g_{m}(x, y)=\sum_{k=1}^{m} \lambda^{-k} \Psi_{k}(x, y) .
$$

By the above definition, we can easily see the following facts.

Lemma 5.2. (1) $\Psi_{m} \geq 0$,

(2) $\operatorname{supp} \Psi_{m} \subset \bigcup_{w \in W_{m-1}} K_{w} \times K_{w}$,

(3) $\left.\Psi_{m}\right|_{V_{m-1} \times V_{m-1}} \equiv 0$,

(4) for some $C>0,\left|\Psi_{m}\right|_{K \times K} \leq C(R / \lambda)^{m}$, for all $m \geq 0$, where $R=$ $\max _{s \in S} r_{s}$.

Definition 5.3. For $(x, y) \in K \times K$, we define

$$
g(x, y)= \begin{cases}\lim _{m \rightarrow \infty} g_{m}(x, y) & \text { if the limit exists and is finite, } \\ \infty & \text { if } g_{m}(x, y) \text { diverges to } \infty \text { as } m \rightarrow \infty\end{cases}
$$

By Lemma 5.2-(1), we can see that $g_{m}(x, y)$ is monotonically increasing as $m \uparrow \infty$. Hence, the preceding definition is well defined. Making use of Lemma 5.2 , we can also verify the following results.

Proposition 5.4. $g_{m}$ converges to $g$, as $m \rightarrow \infty$, uniformly on each compact subset of $K \times K-\{(x, x) \mid x \in K\}$. Furthermore, if $(D, r)$ is regular, then $g_{m}$ converges to $g$, as $m \rightarrow \infty$, uniformly on $K \times K$.

We next state the assumptions on measures under which the Green operator can be defined.

Definition 5.5. A measure $\mu$ is said to be admissible with respect to $(D, r)$ if

(1) $\mu$ is a regular Borel measure on $K$ and $\mu(K)=1$,

(2) $\mu(0)>0$ for each open set $0 \subset K$,

(3) $\mu\left(V_{0}\right)=0$,

(4) $\lim _{m \rightarrow \infty} \int_{K} g_{D}^{m} d \mu<\infty$, where $g_{D}^{m}$ is defined by $g_{D}^{m}(x)=g_{m}(x, x)$.

Remark. Using some usual discussion on measures and integration, we can see that (4) is equivalent to

(4') $g_{D} \in L^{1}(K, \mu)$, where $g_{D}$ is defined by $g_{D}(x)=g(x, x)$.

The assumptions (1)-(3) in the above definition are not so restrictive, for example, they hold for the self-similar measures $\mu_{\eta}$ introduced in $\S 1$. If $(D, r)$ is regular, then (4) becomes trivial because $g_{D}^{m}$ converges to $g_{D}$ uniformly on $K \times K$. In this case, every measure with $(1)-(3)$ is admissible with respect to $(D, r)$. On the other hand, if $(D, r)$ is nonregular, then (4) is not trivial. In this case, however, we can see that 
Proposition 5.6. There exists a self-similar measure $\mu_{\eta}$ which is admissible with respect to $(D, r)$.

Lemma 5.6.1. Let $\mu_{\eta}$ be a self-similar measure.

$$
\text { If } \sum_{s \in S} r_{s} \eta_{s}<\lambda, \text { then } \lim _{m \rightarrow \infty} \int_{K} g_{D}^{m} d \mu<\infty .
$$

Proof. Note that $C=\sup _{x \in K_{w}}\left(-{ }^{t} \psi_{w}(x) X^{-1} \psi_{w}(x)\right)$ is independent of $w \in W_{*}$. By Lemma 1.13, we can see that

$$
\int_{K} \Psi_{m}(x, x) \mu_{\eta}(d x) \leq C \sum_{w \in W_{m-1}} r_{w} \mu_{\eta}\left(K_{w}\right) \leq C\left(\sum_{s \in S} r_{s} \eta_{s}\right)^{m-1} .
$$

Hence,

$$
\int_{K} g_{D}^{m} d \mu \leq C \lambda^{-1} \sum_{k=1}^{m}\left(\lambda^{-1} \sum_{s \in S} r_{s} \eta_{s}\right)^{k-1}
$$

This completes the proof of Lemma 5.6.1.

Proof of Proposition 5.6. By Theorem 4.10, $r_{s}<\lambda$ for some $s \in S$. Hence we can choose $\eta=\left(\eta_{s}\right)_{s \in S}$ so that $\sum_{s \in S} r_{s} \eta_{s}<\lambda$. So, by Lemma 5.6.1, $\mu_{\eta}$ satisfies (4) and therefore $\mu$ is admissible.

Now we are concerned with the Green operator $G_{\mu}$ associated with $(D, r)$. In the rest of this section, $\mu$ is always an admissible measure with respect to $(D, r)$.

Definition 5.7. For $f \in C(K)$ and $x \in K$, we define

$$
\left(G_{\mu}^{m} f\right)(x)=\int_{K} g_{m}(x, y) f(y) \mu(d y),
$$

for all $m \geq 0$, and

$$
\left(G_{\mu} f\right)(x)=\int_{K} g(x, y) f(y) \mu(d y) .
$$

In the course of later discussion, we can show that $G_{\mu}^{m} f$ converges to $G_{\mu} f$ uniformly on $K$ and therefore $G_{\mu} f$ is continuous on $K$. As a result we will obtain the following main theorem of this section.

Theorem 5.8. (1) For any $f \in C(K), G_{\mu} f \in C(K)$ and

$$
\left|G_{\mu} f\right|_{K} \leq\left(\int_{K} g_{D} d \mu\right)|f|_{K}
$$

(2) For any $m \geq 0$ and any $p \in V_{m}$,

$$
\lambda^{m} H_{m, p}\left(G_{\mu} f\right)= \begin{cases}-\int_{K} \psi_{p}^{m} f d \mu & \text { if } p \in V_{m}^{\circ}, \\ -\int_{K}\left(\psi_{p}^{m}-\psi_{p}\right) f d \mu & \text { if } p \in V_{0} .\end{cases}
$$

In the following we prove the above theorem step by step. 
Lemma 5.8.1. For any $w \in W_{m}$,

$$
g\left(F_{w}(x), F_{w}(y)\right)-g_{m}\left(F_{w}(x), F_{w}(y)\right)=\lambda^{-m} r_{w} g(x, y) .
$$

Proof. Recalling the definition of $\psi_{w}$ and $F_{w}$, we can easily see that

$$
\psi_{u} \circ F_{w}= \begin{cases}\psi_{v} & \text { if } u=w \cdot v \\ 0 & \text { otherwise. }\end{cases}
$$

Therefore for $w \in W_{m}$,

$$
\begin{aligned}
g\left(F_{w}(x), F_{w}(y)\right)-g_{m}\left(F_{w}(x), F_{w}(y)\right) & \\
= & -\sum_{i=1}^{\infty} \lambda^{-(m+i)} \sum_{u \in W_{m+i-1}} r_{u}^{t} \psi_{u}\left(F_{w}(x)\right) X^{-1} \psi_{u}\left(F_{w}(y)\right) \\
& =\lambda^{-m} r_{w}\left(-\sum_{i=1}^{\infty} \lambda^{-i} \sum_{v \in W_{i-1}} r_{v}^{t} \psi_{v}(x) X^{-1} \psi_{v}(y)\right) \\
& =\lambda^{-m} r_{w} g(x, y) .
\end{aligned}
$$

Lemma 5.8.2. For any $m \geq 0$ and any $p \in V_{m}$,

$$
\lambda^{m} H_{m, p} g^{x}= \begin{cases}-\psi_{p}^{m}(x) & \text { for } p \in V_{m}^{\circ}, \\ -\psi_{p}^{m}(x)+\psi_{p}(x) & \text { for } p \in V_{0}\end{cases}
$$

where $g^{x}$ is defined by $g^{x}(y)=g(x, y)$.

Proof. Using Lemma 4.8, we can verify that

$$
\lambda^{m} H_{m, p} \psi_{w}=\lambda^{|w|+1} H_{w}^{*} \psi_{p}^{m} .
$$

Hence

$$
\begin{aligned}
\lambda^{m} H_{m, p} g^{x} & =-\sum_{i=1}^{\infty}-\lambda^{-i} \sum_{w \in W_{i-1}} r_{w}{ }^{t} \psi_{w}(x) X^{-1}\left(\lambda^{m} H_{m, p} \psi_{w}\right) \\
& =-\sum_{w \in W .}{ }^{t} \psi_{w}(x) \alpha_{w}\left(\psi_{p}^{m}\right) \\
& =-\psi_{p}^{m}(x)+\left(P_{0} \psi_{p}^{m}\right)(x) .
\end{aligned}
$$

Next, we define $G_{m} \in L\left(V_{m}^{\circ}\right)$ by $\left(G_{m}\right)_{p q}=g(p, q) \quad\left(=g_{m}(p, q)\right)$ for $p$ and $q \in V_{m}^{\circ}$. Then we have

Lemma 5.8.3. $G_{m}=\lambda^{-m}\left(-X_{m}\right)^{-1}$.

Proof. Lemma 5.8.2 implies that, for $p$ and $q \in V_{m}^{\circ},\left(\lambda^{m} X_{m} g^{p}\right)_{q}=-\psi_{p}^{m}(q)$. Hence we have $\lambda^{m} X_{m} G_{m}=-I$, where $I$ is the identity. This implies the required equality.

Lemma 5.8.4. For all $x$ and $y \in K, g(x, y) \leq g(y, y)$.

Proof. We let $x \neq y$ because this lemma is trivial if $x=y$. Choosing $w \in \Sigma$ so that $\pi(w)=y$, then

$$
g_{m}(y, y)=\sum_{p \in B_{[w]_{m}}} g_{m}(p, p)\left(\psi_{p}^{m}(y)\right)^{2} .
$$


Hence

$$
\min _{p \in B_{[w] m}} g_{m}(p, p) \leq g_{m}(y, y) .
$$

And so, we can choose $p_{m} \in B_{[w]_{m}}$ so that

$$
\limsup _{m \rightarrow \infty} g_{m}\left(p_{m}, p_{m}\right) \leq g(y, y) \text {. }
$$

On the other hand, we can choose $q_{m} \in V_{m}$ so that

$$
q_{m} \rightarrow x \text { as } m \rightarrow \infty,
$$

and

$$
\lim _{m \rightarrow \infty} g_{m}\left(q_{m}, p_{m}\right)=g(x, y) .
$$

Now combining Lemma 2.7 and Lemma 5.8.3, we have, for all $m \geq 1$,

$$
g_{m}\left(q_{m}, p_{m}\right) \leq g_{m}\left(p_{m}, p_{m}\right) \text {. }
$$

Letting $m \rightarrow \infty$, we obtain $g(x, y) \leq g(y, y)$ as required.

Lemma 5.8.5. For all $x$ and $y \in K$,

$$
g(x, y)-g_{m}(x, y) \leq g(y, y)-g_{m}(y, y) .
$$

Proof. If $g(x, y)-g_{m}(x, y)>0$, then by Lemma 5.2(2), there exists $w \in W_{m}$ such that $x=F_{w}(\bar{x})$ and $y=F_{w}(\bar{y})$ for some $\bar{x}$ and $\bar{y} \in K$. Hence by Lemma 5.8.1,

$$
g(x, y)-g_{m}(x, y)=\lambda^{-m} r_{w} g(\bar{x}, \bar{y}),
$$

and

$$
g(y, y)-g_{m}(y, y)=\lambda^{-m} r_{w} g(\bar{y}, \bar{y}) .
$$

Therefore by Lemma 5.8.4, we can obtain the required inequality.

Proof of Theorem 5.8. (1) For any $f \in C(K)$, by Lemma 5.8.4 and Lemma 5.8.5, we have, for all $m \geq 0$,

$$
\left|g(x, y) f(y)-g_{m}(x, y) f(y)\right| \leq\left(g_{D}(y)-g_{D}^{m}(y)\right)|f|_{K},
$$

where $g_{0} \equiv 0$. Hence, for all $x \in K,\left(G_{\mu} f\right)(x)$ is a finite value and

$$
\left|\left(G_{\mu} f\right)(x)-\left(G_{\mu}^{m} f\right)(x)\right| \leq\left(\int_{K}\left(g_{D}-g_{D}^{m}\right) d \mu\right)|f|_{K} .
$$

Therefore $G_{\mu}^{m} f$ converges to $G_{\mu} f$ uniformly on $K$, and so $G_{\mu} f \in C(K)$. Also letting $m=0$ in (5.1), we have $\left|G_{\mu} f\right|_{K} \leq\left(\int_{K} g_{D} d \mu\right)|f|_{K}$.

(2) We can easily see that

$$
\lambda^{m} H_{m, p}\left(G_{\mu} f\right)=\int_{K}\left(\lambda^{m} H_{m, p} g^{y}\right) f(y) \mu(d y) .
$$

Hence by Lemma 5.8.2, we have the required results.

\section{THE LAPLACE OPERATOR}

The object of this section is to study the Laplace operator $\Delta_{\mu}$ associated with a harmonic structure $(D, r)$ and an admissible measure $\mu$. In $\S 6$ and $\S 7$, $(D, r)$ is always a harmonic structure with $\lambda=\lambda(D, r)$ and $\mu$ is an admissible measure with respect to $(D, r)$.

The Laplace operator is defined as a limit of difference operators $\Delta_{\mu}^{m}$ as follows. 
Definition 6.1. (1) $\Delta_{\mu}^{m}: l\left(V_{m}\right) \rightarrow l\left(V_{m}^{\circ}\right)$ is defined by, for each $p \in V_{m}^{\circ},\left(\Delta_{\mu}^{m} f\right)_{p}=$ $\lambda^{m} \mu_{m, p}^{-1} H_{m, p} f$, where $\mu_{m, p}=\int_{K} \psi_{p}^{m} d \mu$. When no confusion may occur, we also denote $\Delta_{\mu}^{m} \circ P_{m}: C(K) \rightarrow l\left(V_{m}^{\circ}\right)$ by $\Delta_{\mu}^{m}$.

(2) Let $f$ be a continuous function on $K$. If there exists $\varphi \in C(K)$ such that

$$
\max _{p \in V_{m}^{\circ}}\left|\left(\Delta_{\mu}^{m} f\right)_{p}-\varphi(p)\right| \rightarrow 0 \quad \text { as } m \rightarrow \infty
$$

then we let $\Delta_{\mu} f=\varphi$. The domain of $\Delta_{\mu}$ is denoted by $\mathscr{D}_{\mu}$.

By Theorem 6.9 and Corollary 6.10, we will see that the above definition of the Laplace operator justifies the terminologies such as "harmonic function" or "Green function" in the usual sense.

We next introduce the Neumann derivatives at a point of $V_{0}$.

Definition 6.2. For $f \in C(K)$ and $p \in V_{0}$, we let

$$
(d f)_{p}=\lim _{m \rightarrow \infty}-\lambda^{m} H_{m, p} f,
$$

if the limit of the right-hand side exists and is finite.

As $\mu$ is fixed throughout this section, we often use $\Delta$, and $\mathscr{D}$ instead of $\Delta_{\mu}$ and $\mathscr{D}_{\mu}$.

Lemma 6.3. Let $u \in C(K)$ and $v \in \mathscr{D}$. Then, as $m \rightarrow \infty$,

$$
\lambda^{m} \sum_{p \in V_{m}^{\circ}} u(p) H_{m, p} v \rightarrow \int_{K} u \Delta v d \mu .
$$

Proof. We let

$$
f_{m}(x)=\lambda^{m} \sum_{p \in V_{m}^{\circ}}\left(u(p) \mu_{m, p}^{-1} H_{m, p} v\right) \psi_{p}^{m}(x)
$$

and

$$
f(x)=u(x) \Delta v(x)
$$

Then we may show that, as $m \rightarrow \infty$,

$$
\int_{K} f_{m} d \mu \rightarrow \int_{K} f d \mu
$$

By the definition of $\Delta$, similar discussions as in the proof of Theorem 3.7 imply that, as $m \rightarrow \infty, f_{m}$ converges to $f$ uniformly on each compact set in $K-$ $V_{0}$. Also, the $f_{m}$ 's are equibounded. Therefore, by the Lebesgue convergence theorem, we can prove (6.1).

Applying the last lemma, we can verify that the Neumann derivatives exist for any $f \in \mathscr{D}$.

Lemma 6.4. Let $f \in \mathscr{D}$ and $p \in V_{0}$. Then

$$
(d f)_{p}=-(D f)_{p}+\int_{K} \psi_{p} \Delta f d \mu .
$$

Lemma 6.4.1. For $p \in V_{0}$,

$$
(D f)_{p}=\lambda^{m} H_{m, p} f+\lambda^{m} \sum_{q \in V_{m}^{\circ}} \psi_{p}(q) H_{m, q} f .
$$


Proof. By Lemma 4.9,

$$
T_{m}-{ }^{t} J_{m} X_{m}^{-1} J_{m}=\lambda^{-m} D
$$

Hence,

$$
D f=\lambda^{m}\left(H_{m} f\right)_{V_{0}}-\lambda^{m} \cdot{ }^{t} J_{m} X_{m}^{-1}\left(H_{m} f\right)_{V_{m}^{\circ}} .
$$

On the other hand, $\left(H_{m} \psi_{p}\right)_{V_{m}^{\circ}}=0$ implies $-X_{m}^{-1} J_{m} \mathbf{e}_{p}=\left(\psi_{p}\right)_{V_{m}^{\circ}}$. And so, we have

$$
\left(-X_{m}^{-1} J_{m}\right)_{q p}=\psi_{p}(q) .
$$

Combining (6.2) and (6.3), we can complete the proof.

Proof of Lemma 6.4. Using Lemma 6.3, we have, as $m \rightarrow \infty$,

$$
\lambda^{m} \sum_{q \in V_{m}^{\circ}} \psi_{p}(q) H_{m, q} f \rightarrow \int_{K} \psi_{p} \Delta f d \mu .
$$

Hence by Lemma 6.4.1, we obtain

$$
\lim _{m \rightarrow \infty}-\lambda^{m} H_{m, p} f=-(D f)_{p}+\int_{K} \psi_{p} \Delta f d \mu
$$

as required.

Next we establish Gauss-Green's formula, that is,

Theorem 6.5. Let $u$ and $v \in \mathscr{D}$. Then

(1) $\int_{K}(u \Delta v-v \Delta u) d \mu=\sum_{p \in V_{0}}\left(u(p)(d v)_{p}-v(p)(d u)_{p}\right)$.

(2) $\int_{k} \Delta u d \mu=\sum_{p \in V_{0}}(d u)_{p}$.

To prove Theorem 6.5 , we need some results on symmetric forms $\mathscr{E}_{m}$ on $l\left(V_{m}\right)$.

Definition 6.6. $\mathscr{E}_{m}$ is a symmetric form on $l\left(V_{m}\right)$ defined by

$$
\mathscr{E}_{m}(u, v)=-\lambda^{m}{ }^{t} u H_{m} v \text {. }
$$

For ease of notation, we write, for $u$ and $v \in l\left(V_{*}\right)$,

$$
\mathscr{E}_{m}(u, v)=\mathscr{E}_{m}\left(P_{m} u, P_{m} v\right)
$$

By this manner, we frequently regard $\mathscr{E}_{m}$ as a symmetric form on $l\left(V_{*}\right)$.

By the above definition, we can immediately see that

\section{Lemma 6.7.}

$$
\begin{aligned}
\mathscr{E}_{m}(u, v) & =-\lambda^{m} \sum_{p \in V_{m}} u(p) H_{m, p} v \\
& =\lambda^{m} \sum_{p \in V_{m}} \sum_{q \in V_{m}} h_{p q}^{m}(u(p)-u(q))(v(p)-v(q)),
\end{aligned}
$$

where $h_{p q}^{m}=\left(H_{m}\right)_{p q}$.

Lemma 6.8. Let $u \in C(K)$ and $v \in \mathscr{D}$. Then

$$
\lim _{m \rightarrow \infty} \mathscr{E}_{m}(u, v)=\sum_{p \in V_{0}} u(p)(d v)_{p}-\int_{K} u \Delta v d \mu .
$$


Proof. By Lemma 6.7,

$$
\mathscr{E}_{m}(u, v)=\sum_{p \in V_{0}} u(p)\left(-\lambda^{m} H_{m, p} v\right)-\lambda^{m} \sum_{p \in V_{m}^{\circ}} u(p) H_{m, p} v
$$

Hence Lemma 6.3 and Lemma 6.4 imply the required equality.

Proof of Lemma 6.5. Obviously $\mathscr{E}_{m}(u, v)=\mathscr{E}_{m}(v, u)$. Therefore by Lemma 6.8 , we can prove (1). And letting $u \equiv 1$ in (1), we can prove (2).

Next, we are concerned with the Dirichlet problem of Poisson's equation as follows.

Theorem 6.9. For given $\varphi \in C(K)$ and given $\rho \in l\left(V_{0}\right)$, there exists a unique $f \in \mathscr{D}$ such that

$$
\left\{\begin{array}{l}
\Delta f=\varphi, \\
\left.f\right|_{V_{0}}=\rho,
\end{array}\right.
$$

and this $f$ is given by $f=\sum_{p \in V_{0}} \rho_{p} \psi_{p}-G_{\mu} \varphi$.

Corollary 6.10. $f$ is harmonic if and only if $f \in \mathscr{D}$ and $\Delta f=0$.

For the proof of Theorem 6.8, we need further results on the symmetric forms $\mathscr{E}_{m}$ given in the following.

Definition 6.11. (1) For $w \in W_{*},(\cdot, \cdot)_{w}$ is a symmetric form on $l\left(V_{*}\right)$ defined by

$$
(u, v)_{w}=-r_{w}^{-1} \cdot{ }^{t} \alpha_{w}(u) X \alpha_{w}(v) .
$$

(2) $\Phi_{m}(\cdot, \cdot)$ is a symmetric form on $l\left(V_{*}\right)$ defined by

$$
\left\{\begin{array}{l}
\boldsymbol{\Phi}_{m}(u, v)=\sum_{w \in W_{m-1}}(u, v)_{w} \text { for } m \geq 1 \\
\Phi_{0}(u, v)=\mathscr{E}_{0}(u, v)
\end{array}\right.
$$

Lemma 6.12. $(f, f)_{w} \geq 0$ and the equality holds if and only if $\alpha_{w}(f)=0$.

Proof. Using Lemma 2.7, we can see that $-X$ is positive definite. This implies Lemma 6.12.

The following is a remarkable fact about the symmetric forms $\mathscr{E}_{m}$. It is a key result for studying the Dirichlet form $\mathscr{E}$, the limit of $\mathscr{E}_{m}$ in $\S 7$.

Lemma 6.13. For $m \geq 0$,

$$
\mathscr{E}_{m}(u, v)=\sum_{k=0}^{m} \lambda^{k} \Phi_{k}(u, v) .
$$

Lemma 6.13.1. For each $w \in W_{*}$, let $\langle u, v\rangle_{w}=-r_{w}^{-1}{ }^{t} u^{b} D v^{b}$. Then

$$
\mathscr{E}_{m}(u, v)=\lambda^{m} \sum_{w \in W_{m}}\langle u, v\rangle_{w}
$$

Proof. Obvious by the definition of $H_{m}$.

Lemma 6.13.2. For each $w \in W_{*}$,

$$
\sum_{s \in S}\langle u, v\rangle_{w s}-\lambda^{-1}\langle u, v\rangle_{w}=(u, v)_{w}
$$


Proof. First recalling

$$
\alpha_{w}(f)=r_{w}^{-1} X H_{w}^{*} f=r_{w}^{-1} X^{-1}\left(\begin{array}{ll}
J & X
\end{array}\right)\left(\begin{array}{c}
f_{w}^{b} \\
f_{w}^{s}
\end{array}\right),
$$

then we have

$$
(u, v)_{w}=-r_{w}^{-1}\left({ }^{t} u_{w}^{b}{ }^{t} u_{w}^{s}\right)\left(\begin{array}{cc}
{ }^{t} J X^{-1} J & { }^{t} J \\
J & X
\end{array}\right)\left(\begin{array}{c}
v_{w}^{b} \\
v_{w}^{s}
\end{array}\right)
$$

We also obtain

$$
\langle u, v\rangle_{w}=-r_{w}^{-1}\left({ }^{t} u_{w}^{b}{ }^{t} u_{w}^{s}\right)\left(\begin{array}{cc}
D & 0 \\
0 & 0
\end{array}\right)\left(\begin{array}{c}
v_{w}^{b} \\
v_{w}^{s}
\end{array}\right)
$$

and

$$
\sum_{s \in S}\langle u, v\rangle_{w s}=-r_{w}^{-1}\left({ }^{t} u_{w}^{b}{ }^{t} u_{w}^{s}\right)\left(\begin{array}{cc}
T & { }^{t} J \\
J & X
\end{array}\right)\left(\begin{array}{c}
v_{w}^{b} \\
v_{w}^{s}
\end{array}\right) .
$$

Now since $(D, r)$ is a harmonic structure, we have $T-{ }^{t} J X^{-1} J=\lambda^{-1} D$. This together with (6.5), (6.6), and (6.7) completes the proof of Lemma 6.13.2.

Proof of Lemma 6.13. Using Lemma 6.13.2, we see that

$$
\lambda^{m} \sum_{w \in W_{m-1}} \sum_{s \in S}\langle u, v\rangle_{w s}-\lambda^{m-1} \sum_{u \in W_{m-1}}\langle u, v\rangle_{w}=\lambda^{m} \Phi_{m}(u, v) .
$$

Applying Lemma 6.13.1, this implies

$$
\mathscr{E}_{m}(u, v)-\mathscr{E}_{m-1}(u, v)=\lambda^{m} \Phi_{m}(u, v)
$$

Hence we can obtain the required result.

Corollary 6.14. For each $m \geq 0, \mathscr{E}_{m}(u, u) \leq \mathscr{E}_{m+1}(u, u)$. And $\mathscr{E}_{m}(u, u)=$ $\lim _{k \rightarrow \infty} \mathscr{E}_{k}(u, u)$ if and only if $u$ is m-harmonic.

Proof. By Lemma 6.12, $\mathscr{E}_{m+1}(u, u)-\mathscr{E}_{m}(u, u)=\lambda^{m+1} \Phi_{m+1}(u, u) \geq 0$. And we can see that $\mathscr{E}_{m}(u, u)=\lim _{k \rightarrow \infty} \mathscr{E}_{k}(u, u)$ if and only if $\Phi_{n}(u, u)=0$ for all $n \geq m$. Hence by Lemma $6.12, \alpha_{w}(f)=0$ for $w \in W_{*}-W_{m-1}$. This is equivalent to the statement that $f$ is $m$-harmonic.

Proof of Theorem 6.9. For the uniqueness, let $\varphi \equiv 0$ and $\rho \equiv 0$ and show that $f \equiv 0$. If $\left.f\right|_{V_{0}} \equiv 0$ and $\Delta f \equiv 0$, then Lemma 6.8 implies that

$$
\lim _{m \rightarrow \infty} \mathscr{E}_{m}(f, f)=0 \text {. }
$$

Using Corollary 6.14, we can see that $f$ is harmonic. Therefore by $\left.f\right|_{V_{0}} \equiv 0$, we obtain $f \equiv 0$. Now, we may prove that if $f=-G_{\mu} \varphi$, then $\Delta f=\varphi$. By Theorem 5.8,

$$
\mu_{m, p}^{-1} \lambda^{m} H_{m, p} f=\mu_{m, p}^{-1} \int_{K} \psi_{p}^{m} \varphi d \mu
$$

Noting that $\varphi$ is uniformly continuous on $K$ and

$$
\max _{p \in V_{m}^{\circ}}\left(\text { diameter of supp } \psi_{p}^{m}\right) \rightarrow 0 \text { as } m \rightarrow \infty,
$$

we can verify that

$$
\max _{p \in V_{m}^{\circ}}\left|\lambda^{m} \mu_{m, p}^{-1} H_{m, p} f-\varphi(p)\right| \rightarrow 0 \quad \text { as } m \rightarrow \infty .
$$

Therefore $\Delta f=\varphi$. 
Finally we remark that the Dirichlet problem for Poisson's equation (6.4) is equivalent to the following infinite system of finite difference equations on $V_{*}$,

$$
\left\{\begin{array}{l}
\left.f\right|_{V_{0}}=\rho, \\
\lambda^{i(p)} H_{p}^{*} f=\int_{K} \psi_{p} \varphi d \mu \text { for each } p \in V_{*} .
\end{array}\right.
$$

Such an equivalence was obtained in [15] and [10] for simple cases.

\section{DiRICHLET FORM}

In this section, we construct a Dirichlet form $\mathscr{E}$ associated with a regular harmonic structure as a limit of symmetric forms $\mathscr{E}_{m}$ on $l\left(V_{m}\right)$. In the present paper, we will not introduce the detailed study on Dirichlet forms. The reader may refer to Fukushima [4] for the definition and the results on Dirichlet forms.

Throughout this section, we fix a regular harmonic structure $(D, r)$ with $\lambda=\lambda(D, r)$ and an admissible measure $\mu$ with respect to $(D, r)$. We remark that under a regular harmonic structure, Green's function $g$ is continuous on $K \times K$ and every self-similar measure is admissible.

By Corollary 6.14 , we can see that $\lim _{m \rightarrow \infty} \mathscr{E}_{m}(f, f)$ exists for each $f \in$ $l\left(V_{*}\right)$ if we let the value of limit be $\infty$. We define a symmetric form $\mathscr{E}$ as follows.

Definition 7.1. A subspace $\mathscr{F} \subset l\left(V_{*}\right)$ is defined by

$$
\mathscr{F}=\left\{f \mid f \in l\left(V_{*}\right) \text { and } \lim _{m \rightarrow \infty} \mathscr{E}_{m}(f, f)<\infty\right\} .
$$

And a symmetric form $\mathscr{E}$ on $\mathscr{F}$ is defined by $\mathscr{E}(u, v)=\lim _{m \rightarrow \infty} \mathscr{E}_{m}(u, v)$.

Obviously, $\mathscr{E}$ is nonnegative definite and $\mathscr{E}_{m}(u, v)=\mathscr{E}\left(P_{m} u, P_{m} v\right)$. Now, Corollary 6.14 shows that

Proposition 7.2. $l\left(V_{m}\right) \subset \mathscr{F}$ for all $m \geq 0$. And $f \in l\left(V_{m}\right)$ if and only if $\mathscr{E}_{m}(f, f)=\mathscr{E}(f, f)$.

Also by Lemma 6.8 , we can obtain

Proposition 7.3. Let $\mu$ be admissible. Then $\mathscr{D}_{\mu} \subset \mathscr{F}$ and

$$
\mathscr{E}(u, v)=\sum_{p \in V_{0}} u(p)(d v)_{p}-\int_{K} u \Delta_{\mu} v d \mu,
$$

for $u \in C(K) \cap \mathscr{F}$ and $v \in \mathscr{D}_{\mu}$.

Here we state the main results of this section.

Theorem 7.4. Let $\mu$ be admissible. Then,

(1) $\mathscr{F} \subset C(K)$,

(2) $(\mathscr{F}, \mathscr{E})$ is a regular local Dirichlet space on $L^{2}(K, \mu)$,

(3) $\mathscr{E}\left(f, g^{x}\right)=f(x)-\left(P_{0} f\right)(x)$ for all $f \in \mathscr{F}$ and all $x \in K$,

(4) $(\mathscr{F}, \mathscr{E})$ is the minimal closed extension of $\left(\mathscr{D}_{\mu}, \mathscr{E}\right)$,

(5) $\mathscr{D}_{\mu}$ is dense in $C(K)$. 
In the following, we prove the above theorem step by step.

For ease of notation, we let

$$
[f]_{m}=\sqrt{\mathscr{E}_{m}(f, f)} \text { and }[f]=\sqrt{\mathscr{E}(f, f)} .
$$

First we observe some facts immediately obtained by the preceding definitions.

Lemma 7.4.1. Let $u$ and $v \in \mathscr{F}$. Then,

(1) $\left[P_{m} u\right]=[u]_{m}$,

(2) $[u]_{m} \uparrow[u]$ as $m \uparrow \infty$,

(3) $\left[P_{m} u-P_{n} u\right]^{2}=[u]_{m}^{2}-[u]_{n}^{2}$ for $m>n$,

(4) $|\mathscr{E}(u, v)| \leq[u][v]$.

Lemma 7.4.2. Let $f \in l\left(V_{*}\right)$ and $x \in K$. Then

$$
\mathscr{E}_{m}\left(f, g^{x}\right)=\left(P_{m} f\right)(x)-\left(P_{0} f\right)(x) .
$$

Proof. By the definition of $g$ and $\alpha_{w}$, we can see that

$$
\alpha_{w}\left(g^{x}\right)=-\lambda^{-(|w|+1)} r_{w} X^{-1} \psi_{w}(x) .
$$

Hence $\left(f, g^{x}\right)_{w}=\lambda^{-(|w|+1)} \cdot{ }^{t} \alpha_{w}(f) \psi_{w}(x)$. Therefore by Lemma 6.13,

$$
\mathscr{E}_{m}\left(f, g^{x}\right)=\sum_{k=1}^{m} \lambda^{k} \Phi_{k}\left(f, g^{x}\right)=\sum_{w \in W_{m}}{ }^{t} \alpha_{w}(f) \psi_{w}(x) .
$$

This implies the required result.

It follows immediately from the last lemma that

Lemma 7.4.3. For any $x \in K, g^{x} \subset \mathscr{F}$, and $\mathscr{E}\left(g^{x}, g^{x}\right)=g(x, x)$.

Lemma 7.4.4. If $f \in \mathscr{F}$, then $f \in C(K)$ and for all $x \in K$,

$$
\mathscr{E}\left(f, g^{x}\right)=f(x)-\left(P_{0} f\right)(x) .
$$

Proof. By Lemma 3.7, we may show that $P_{m} f$ converges uniformly on $K$. Using Lemma 7.4.1(4), we have

$$
\left|\mathscr{E}\left(P_{m} f-P_{n} f, g^{x}\right)\right| \leq\left[P_{m} f-P_{n} f\right]\left[g^{x}\right],
$$

for all $x \in K$ and $m>n$. Applying Lemma 7.4.1(3), we obtain

$$
\left|P_{m} f(x)-P_{n} f(x)\right| \leq\left([f]_{m}^{2}-[f]_{n}^{2}\right)^{1 / 2} g_{D}(x) .
$$

Hence

$$
\left|P_{m} f-P_{m} f\right|_{K} \leq\left([f]_{m}^{2}-[f]_{n}^{2}\right)^{1 / 2}\left|g_{D}\right|_{K} .
$$

Again using Lemma 7.4.1(2), this implies that $P_{m} f$ converges uniformly on $K$.

We let, for $u$ and $v \in \mathscr{F}$,

$$
\mathscr{E}^{*}(u, v)=\mathscr{E}(u, v)+\int_{K} u v d \mu .
$$

Also, $\mathscr{F}_{0}$ is defined by

$$
\mathscr{F}_{0}=\left\{f \mid f \in \mathscr{F} \text { and } P_{0} f \equiv 0\right\} .
$$


Lemma 7.4.5. $\mathscr{F}_{0}$ is a Hilbert space with the inner product $\mathscr{E} *$.

Proof. By Lemma 7.4.4, if $f \in \mathscr{F}_{0}$, then

$$
|f|_{K} \leq[f]\left|g_{D}\right|_{K} .
$$

Hence there exists $C>0$ such that for all $f \in \mathscr{F}_{0}$,

$$
[f] \leq\left(\mathscr{E}^{*}(f, f)\right)^{1 / 2} \leq C[f] .
$$

Therefore, we may show that $\mathscr{F}_{0}$ is a Hilbert space by the inner product $\mathscr{E}$. Let $\left\{f_{m}\right\}_{m \geq 1}$ be a Cauchy sequence in $\left(\mathscr{F}_{0}, \mathscr{E}\right)$, that is, there exists $\left\{\varepsilon_{m}\right\}_{m \geq 1}$ such that $\lim _{m \rightarrow \infty} \varepsilon_{m}=0$ and $\left[f_{m}-f_{n}\right]<\varepsilon_{n}$ for all $m \geq n$. Then, for each $k \geq 0$, $\left\{P_{k} f_{m}\right\}_{m \geq 1}$ is a Cauchy sequence in $l\left(V_{k}\right)$ and hence there exists $f \in l\left(V_{*}\right)$ satisfying that, for each $k \geq 0, P_{k} f_{m} \rightarrow P_{k} f$ as $m \rightarrow \infty$ in $l\left(V_{k}\right)$. Now, for $m \geq n$,

$$
\left[P_{k} f_{m}-P_{k} f_{n}\right]=\left[f_{m}-f_{n}\right]_{k} \leq\left[f_{m}-f_{n}\right]<\varepsilon_{n} .
$$

Hence, letting $m \rightarrow \infty$, we obtain $\left[P_{k} f-P_{k} f_{n}\right]=\left[f-f_{n}\right]_{k} \leq \varepsilon_{n}$. Again letting $k \rightarrow \infty$, we can see that $f \in \mathscr{F}_{0}$ and $\left[f-f_{n}\right] \leq \varepsilon_{n}$. Hence $f_{m}$ converges to $f$ in $(\mathscr{F}, \mathscr{E})$.

Lemma 7.4.6. $\mathscr{F}$ is a Hilbert space with the inner product $\mathscr{E}^{*}$.

Proof. Note that $\mathscr{F}=\mathscr{F}_{0} \oplus l\left(V_{0}\right)$ and use Lemma 7.4.5.

Proof of Theorem 7.4. We have shown (1) and (3) in the course of the above arguments.

(2) By Lemma 7.4.6, we see that $\mathscr{E}$ is a closed form on $L^{2}(K, \mu)$.

(i) Regularity. Recalling Proposition 7.2, we can verify that $\bigcup_{m \geq 0} l\left(V_{m}\right)$ is a core.

(ii) Markov property. For $u \in \mathscr{F}$, we define $\bar{u} \in l\left(V_{*}\right)$ by

$$
\bar{u}(x)= \begin{cases}u(x) & \text { if } 0<u(x)<1 \\ 1 & \text { if } 1 \leq u(x) \\ 0 & \text { if } u(x) \leq 0\end{cases}
$$

Then, for each $p$ and $q \in V_{*}$,

$$
(\bar{u}(p)-\bar{u}(q))^{2} \leq(u(p)-u(q))^{2} .
$$

Using Lemma 6.7 , we have $\mathscr{E}_{m}(\bar{u}, \bar{u}) \leq \mathscr{E}_{m}(u, u)$. Therefore $\bar{u} \in \mathscr{F}$ and $\mathscr{E}(\bar{u}, \bar{u}) \leq \mathscr{E}(u, u)$. Thus, $\mathscr{E}$ is stable under the unit contraction.

(iii) Local property. Let $f_{1}$ and $f_{2} \in \mathscr{F}$ with $\operatorname{supp} f_{1} \cap \operatorname{supp} f_{2}=\varnothing$. Then, for sufficiently large $m$, there exists $U_{i} \subset W_{m}$ such that

$$
\operatorname{supp} f_{i} \subset \bigcup_{w \in U_{i}} K_{w} \text { and }\left(\bigcup_{w \in U_{1}} K_{w}\right) \cap\left(\bigcup_{w \in U_{2}} K_{w}\right)=\varnothing .
$$

Hence using Lemma 6.7, we can see that $\mathscr{E}\left(f_{1}, f_{2}\right)=0$.

Thus we have shown that $(\mathscr{F}, \mathscr{E})$ is a regular local Dirichlet space on $L^{2}(K, \mu)$.

(4) Since $\mathscr{F}=\mathscr{F} \oplus l\left(V_{0}\right)$ and $l\left(V_{0}\right) \subset \mathscr{D}$, we may prove that $\mathscr{D}_{0}=\mathscr{F}_{0} \cap \mathscr{D}$ is a dense subset of the Hilbert space $(\mathscr{F}, \mathscr{E})$. For $p \in V_{*}$, we let $\varphi_{p}^{m}=\mu_{m, p}^{-1} \psi_{p}^{m}$. 
Then, using Proposition 7.3, Lemma 7.4.3, and Lemma 7.4.4,

$$
\begin{aligned}
{\left[G_{\mu} \varphi_{p}^{m}-g^{p}\right]^{2}=} & {\left[G_{\mu} \varphi_{p}^{m}\right]^{2}-2 \mathscr{E}\left(G_{\mu} \varphi_{p}^{m}, g^{p}\right)+g(p, p) } \\
= & \int_{K \times K} g(x, y) \varphi_{p}^{m}(x) \varphi_{p}^{m}(y) \mu(d y) \mu(d x) \\
& -2 \int_{K} g(p, y) \varphi_{p}^{m}(y) \mu(d y)+g(p, p) .
\end{aligned}
$$

By a similar discussion as in the proof of Theorem 6.9 , we can see that

$$
\left[G_{\mu} \varphi_{p}^{m}-g^{p}\right] \rightarrow 0 \text { as } m \rightarrow \infty .
$$

On the other hand, by Lemma 5.8.2, $-\sum_{q \in V_{m}} h_{p q}^{m} g^{q}=\psi_{p}^{m}$. Hence,

$$
\left[G_{\mu}\left(-\sum_{q \in V_{m}} h_{p q}^{m} \varphi_{q}^{m}\right)-\psi_{p}^{m}\right] \rightarrow 0 \quad \text { as } m \rightarrow \infty .
$$

Therefore, $\operatorname{cl}\left(\mathscr{D}_{0}\right)$ in $\left(\mathscr{F}_{0}, \mathscr{E}\right)$ contains every $\psi_{p}^{m}$. Note that $\bigcup_{m \geq 1} l\left(V_{m}\right)$ is dense in $\left(\mathscr{F}_{0}, \mathscr{E}\right)$, we can conclude that $\mathscr{D}_{0}$ is dense in $(\mathscr{F}, \mathscr{E})$.

(5) Note that $\bigcup_{m \geq 0} l\left(V_{m}\right)$ is dense in $C(K)$. Using (7.1), we can show that $\mathscr{D}$ is dense in $C(K)$.

\section{EXAMPLES}

This section is devoted to five examples of p.c.f. self-similar sets and harmonic structures on them.

First in item (I), we describe $S, C, q$ for each $q \in \pi^{-1}(C), P$ and $p$ for each $p \in \pi^{-1}(P)$. According to the discussion in Appendix A, item (I) gives complete information about $\pi$ and determines the equivalence class of the selfsimilar structure.

Secondly in item (II), we give some harmonic structures.

Finally in item (III), we describe the concrete self-similar structure, where each self-similar set is immersed in $\mathbb{C}$ and each $F_{S}$ is the contracting similitude of $\mathbb{C}$.

In each example, we use $\dot{s}$ for $s \in S$ which is defined by $(\dot{s})_{i}=s$ for all $i \geq 1$.

Example 8.1. Interval.

$$
\text { (II) } \begin{aligned}
S & =\{1,2\} . \\
C & =\{1 \dot{2}, 2 \mathrm{i}\}, q=\pi(1 \dot{2})=\pi(2 \dot{\mathrm{i}}), \\
P & =\{\dot{\mathrm{i}}, \dot{2}\}, p_{1}=\pi(\mathrm{i}), p_{2}=\pi(\dot{2}) . \\
\text { (II) } D & =\left(\begin{array}{cc}
-1 & 1 \\
1 & -1
\end{array}\right), \\
r & =(\alpha, 1-\alpha) \text { for } 0<\alpha<1, \lambda=1 .
\end{aligned}
$$

When $\alpha=\frac{1}{2},\left(\mathscr{F}, \mathscr{E}^{*}\right)$ equals the $H^{1}$-Sobolev spaces. 
CALCULUS ON SELF-SIMILAR SETS

749

(III) $p_{1}=0, p_{2}=1, q=\frac{1}{2}$,

$$
F_{1}(z)=\frac{1}{2} z, F_{2}(z)=\frac{1}{2} z-\frac{1}{2} \text {. }
$$

$$
p_{1} \stackrel{\bullet=0}{q=\frac{1}{2}} \quad p_{2}=1
$$

FIGURE 1

Example 8.2. Sierpinski gasket.

(I)

$$
\begin{aligned}
& S=\{1,2,3\} \\
& C=\{2 \dot{3}, 3 \dot{2}, 1 \dot{3}, 3 \dot{1}, 1 \dot{2}, 2 \dot{\mathrm{i}}\}, q_{1}=\pi(1 \dot{\mathrm{i}})=\pi(2 \dot{\mathrm{i}}), \\
& q_{2}=\pi(1 \dot{3})=\pi(3 \dot{\mathrm{i}}), q_{3}=\pi(1 \dot{2})=\pi(2 \dot{\mathrm{i}}) . \\
& P=\{\dot{1}, \dot{2}, \dot{3}\}, p_{s}=\pi(\dot{s}) \text { for } s=1,2,3 .
\end{aligned}
$$

(II) $D=\left(\begin{array}{ccc}-2 & 1 & 1 \\ 1 & -2 & 1 \\ 1 & 1 & -2\end{array}\right)$,

$$
r=(1,1,1), \lambda=\frac{5}{3} \text {. }
$$

(III) $p_{1}, p_{2}$, and $p_{3}$ are the vertices of regular triangle.

$$
q_{1}=\frac{1}{2}\left(p_{2} p_{3}\right), q_{2}=\frac{1}{2}\left(p_{1} p_{3}\right), q_{3}=\frac{1}{2}\left(p_{1} p_{2}\right),
$$

where $\frac{1}{2}\left(p_{i} p_{j}\right)$ is the midpoint of $p_{i}$ and $p_{j}$.

$$
F_{s}(z)=\frac{1}{2}\left(z-p_{s}\right)+p_{s} \text {. }
$$

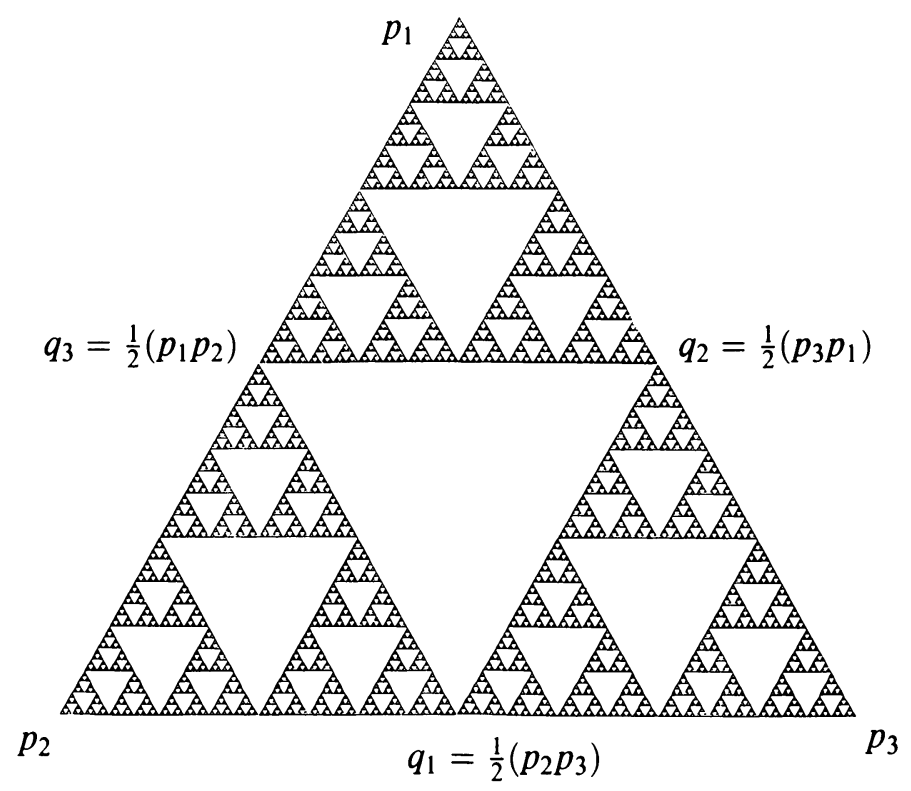

FIGURE 2 


\section{Example 8.3.}

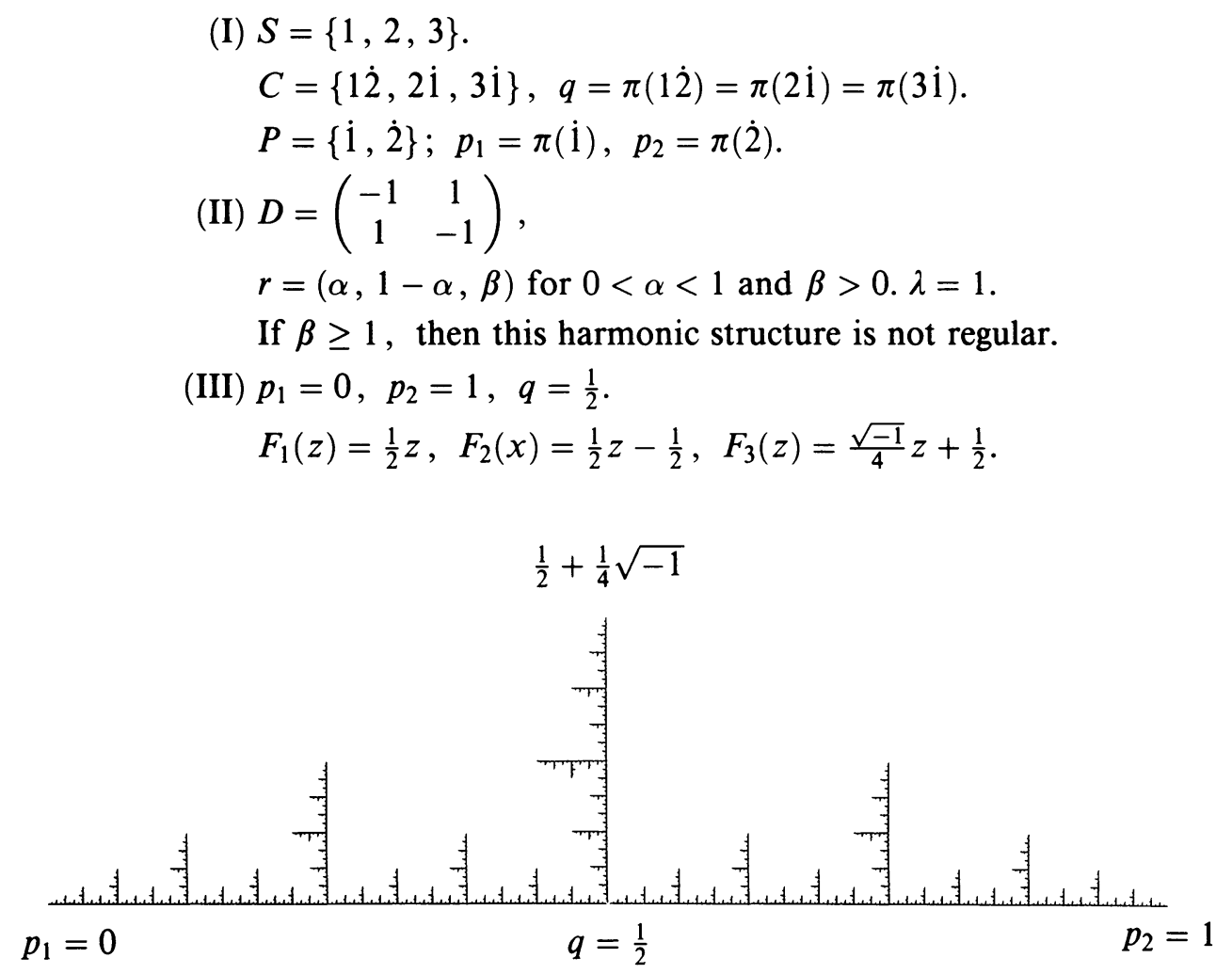

Figure 3

Example 8.4. Hata's tree-like set.

This tree-like self-similar set was found by Hata [5].

(I) $S=\{1,2\}$.

$C=\{11 \dot{2}, 2 \mathrm{i}\}, q=\pi(11 \dot{2})=\pi(2 \mathrm{i})$.

$P=\{\dot{1}, \dot{2}, 1 \dot{2}\} ; p_{s}=\pi(\dot{s})$ for $s \in S, p_{3}=\pi(1 \dot{2})$.

This self-similar structure does not satisfy (1).

(II) $D=\left(\begin{array}{ccc}-\left(1+\alpha^{-1}\right) & 1 & \alpha^{-1} \\ 1 & -1 & 0 \\ \alpha^{-1} & 0 & -\alpha^{-1}\end{array}\right)$,

$r=\left(\alpha, 1-\alpha^{2}\right)$ for $0<\alpha<1, \lambda=1$.

(III) $p_{1}=0, p_{2}=1, p_{3}=\beta, q=|\beta|^{2}$.

$F_{1}(z)=\beta \bar{z}, \quad F_{2}(z)=\left(1-|\beta|^{2}\right) \bar{z}+|\beta|^{2}$,

where $|\beta|<1,|1-\beta|<1$ and $\operatorname{Im} \beta \neq 0$.

In Figure $4, \beta=0.4-0.3 \sqrt{-1}$. 


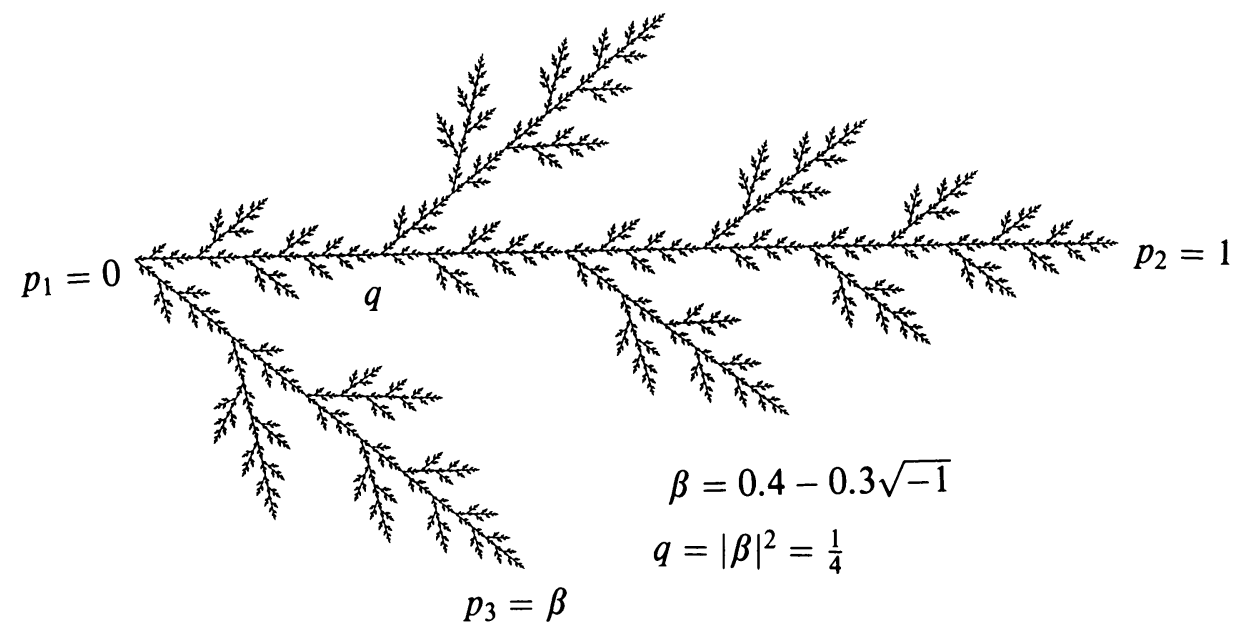

FigURE 4

Example 8.5. Nested fractal.

$S=\{1,2, \ldots, N\}$. Each $F_{s}$ is a $\nu$-similitude of $\mathbb{R}^{n}$, that is, $\mid F_{s}(x)-$ $F_{s}(y)|=\nu| x-y \mid$ for all $x, y \in \mathbb{R}^{n}$.

$V_{0}$ is the subset of the fixed points of $F_{s}$ 's. An element of $V_{0}$ is called an essential fixed point by Lindstrøm.

Let $P$ be the matrix of invariant transition probabilities of random walk on $V_{0}$ given by Lindstrøm. Then $(P-I,(\nu, \ldots, \nu))$ is a regular harmonic structure on the nested fractal, where $I$ is the identity matrix. We conjecture that our Dirichlet forms and the Dirichlet forms associated with the diffusion processes given by Lindstrøm [13] are the same.

\section{APPENDIX A}

We begin with some obvious facts.

Lemma A.1. Let $\left(K, S,\left\{F_{s}\right\}_{s \in S}\right)$ be a p.c.f. self-similar structure. Then,

(1) $V_{1}=\bigcup_{s \in S} F_{s}\left(V_{0}\right)$.

(2) If $V_{0} \neq \varnothing$, then for all $s \in S, F_{s}\left(V_{0}\right)-V_{0} \neq \varnothing$.

Definition A.2. Let $S, V$, and $U$ be finite sets with $V \supset U$ and let $G_{s}: U \rightarrow V$ be an injection for each $s \in S$. Then $\mathscr{A}=\left(S, V, U,\left\{G_{s}\right\}_{s \in S}\right)$ is called an ancestor if

(1) $V=\bigcup_{s \in S} G_{s}(U)$.

(2) If $U \neq \varnothing$, then for all $s \in S, G_{s}(U)-U \neq \varnothing$.

In particular, for a p.c.f. self-similar structure $\mathscr{L}=\left(K, S,\left\{F_{s}\right\}_{s \in S}\right), \mathscr{A}(\mathscr{L})=$ $\left(S, V_{1}, V_{0},\left\{\left.F_{s}\right|_{V_{0}}\right\}_{s \in S}\right)$ is called the ancestor of $\mathscr{L}$.

By the following procedure, we can construct a p.c.f. self-similar structure from a given ancestor.

Definition A.3. Let $\mathscr{A}=\left(S, V, U,\left\{G_{s}\right\}_{s \in S}\right)$ be an ancestor. Then, for each $x \in V, \mathscr{A}_{x} \subset \Sigma(S)$ is the collection of all $w$ satisfying that there exists 
$\left\{x_{i}\right\}_{i \geq 1} \subset U$ such that $G_{w_{1}}\left(x_{1}\right)=x$ and $G_{w_{i}}\left(x_{i}\right)=x_{i-1}$ for all $i \geq 2$. Further a relation $\tilde{\mathscr{A}}$ on $\Sigma(S)$ is defined by

$w \underset{\mathscr{A}}{\sim} v$ if and only if $w=v$ or there exist $x \in V, w_{*}, v_{*} \in \mathscr{A}_{x}$ and $u \in W_{*}(S)$ satisfying $w=u \cdot w_{*}$ and $v=u \cdot v_{*}$.

Lemma A.4. Let $\mathscr{A}=\left(S, V, U,\left\{G_{s}\right\}_{s \in S}\right)$ be an ancestor. Then,

(1) $\tilde{\mathscr{A}}$ is an equivalence relation on $\Sigma(S)$.

(2) If $w \tilde{\mathscr{A}} v$, then $s \cdot w \tilde{\mathscr{A}} s \cdot v$ for all $s \in S$.

(3) $K_{\mathscr{A}}=\Sigma(S) / \widetilde{\mathscr{A}}$ equipped with the quotient topology from $\Sigma(S)$ is metrizable.

(4) For each $s \in S, F_{s}^{\mathscr{A}}=\pi_{\mathscr{A}} \circ S \circ \pi_{\mathscr{A}}^{-1}$ is a well-defined continuous injection, where $\pi_{\mathscr{A}}: \Sigma(S) \rightarrow K_{\mathscr{A}}$ is the natural projection.

(4) of the last lemma follows from results in Kameyama [9]. The other parts follow immediately from Definition A.3.

Theorem A.5. Let $\mathscr{A}=\left(S, V, U,\left\{G_{s}\right\}_{s \in S}\right)$ be an ancestor. Then $\mathscr{L}(\mathscr{A})=$ $\left(K_{\mathscr{A}}, S,\left\{F_{S}^{\mathscr{A}}\right\}_{s \in S}\right)$ is a p.c.f. self-similar structure.

It is natural to expect that $\mathscr{L}(\mathscr{A}(\mathscr{L})) \simeq \mathscr{L}$ for every p.c.f. self-similar structure $\mathscr{L}$. Before stating the results, we may give the exact meaning of " $\simeq$.

Definition A.6. Let $\mathscr{L}=\left(K, S,\left\{F_{s}\right\}_{s \in S}\right)$ and $\mathscr{K}=\left(L, T,\left\{G_{t}\right\}_{t \in T}\right)$ be two self-similar structures. Then, $\mathscr{L} \leq \mathscr{K}$ if and only if there exist a bijection $\tau: S \rightarrow T$ and a continuous surjection $h: K \rightarrow L$ such that $G_{\tau(s)} \circ h=h \circ F_{s}$ for any $s \in S . \mathscr{L} \simeq \mathscr{K}$ if and only if $\mathscr{L} \geq \mathscr{K}$ and $\mathscr{L} \leq \mathscr{K}$.

Definition A.7. Let $\mathscr{A}=\left(S, V, U,\left\{G_{s}\right\}_{s \in S}\right)$ and $\mathscr{B}=\left(T, W, X,\left\{H_{t}\right\}_{t \in T}\right)$ be two ancestors. Then $\mathscr{A} \leq \mathscr{B}$ if and only if there exist a bijection $\tau: S \rightarrow T$ and an injection $h: V \rightarrow W$ such that $h(U) \subset X$ and $H_{\tau(s)} \circ h=h \circ G_{s}$ for each $s \in S$.

Finally, we can give the main result of Appendix A.

Theorem A.8. Let $\mathscr{A}$ and $\mathscr{B}$ be ancestors and let $\mathscr{L}$ and $\mathscr{K}$ be p.c.f. selfsimilar structures. Then,

(1) If $\mathscr{L} \leq \mathscr{K}$, then $\mathscr{A}(\mathscr{L}) \leq \mathscr{A}(\mathscr{K})$.

(2) If $\mathscr{A} \leq \mathscr{B}$, then $\mathscr{L}(\mathscr{A}) \leq \mathscr{L}(\mathscr{B})$.

(3) $\mathscr{L}(\mathscr{A}(\mathscr{L})) \simeq \mathscr{L}$.

(4) $\mathscr{A}(\mathscr{L}(\mathscr{A})) \leq \mathscr{A}$.

The proof is straightforward and left to the reader.

APPENDIX B

This appendix is devoted to the proof of Theorem 2.6.

Theorem 2.6. Suppose that

$\#\left(B_{s} \cap V_{0}\right) \leq 1$ for each $s \in S$. 
Then, for each $\rho \in l\left(V_{0}\right)$, there exist a unique quasi-harmonic function $f$ with $\left.f\right|_{V_{0}}=\rho$.

In the following, we let $V_{0}=\{1,2, \ldots, M\}$ and $l\left(V_{0}\right)=\mathbb{R}^{M}$ for ease of notation.

Before starting the discussion, we summarize the results of the heuristic arguments in $\S 2$.

Lemma B.1. Let $A_{s}=R_{s}\left(\begin{array}{c}I \\ -X^{-1 J}\end{array}\right)$ for each $s \in S$. Then $A_{s}$ is a stochastic matrix and for every quasi-harmonic function $f$ and any $w \in W_{*}$,

$$
(f)_{w}^{b}=A_{w}(f)_{V_{0}},
$$

where $A_{w}=A_{w_{m}} A_{w_{m-1}} \cdots A_{w_{1}}$ if $w=w_{1} w_{2} \cdots w_{m}$.

Now, for $f \in l\left(V_{0}\right)$, we define $v(f)=\max _{1 \leq i, j \leq M}\left|f_{i}-f_{j}\right|$.

Lemma B.2. For any $f \in l\left(V_{0}\right)$ with $v(f) \neq 0, v\left(A_{s} f\right)<v(f)$ for each $s$.

Proof. As $v(f) \neq 0$, we have

$$
L_{1}=\max _{j \in V_{0}} f_{j}>L_{2}=\min _{j \in V_{0}} f_{j} .
$$

We extend $f$ to a function on $V_{1}$ by $\left.f\right|_{V_{1}^{\circ}}=-X^{-1} J f$. Then, $A_{s} f=R_{s} f$ and, for all $s \in S$,

$$
L_{2} \leq \min _{j \in B_{s}} f_{j} \leq \max _{j \in B_{s}} f_{j} \leq L_{1} .
$$

Now, if $v(f)=v\left(A_{s}\right)$, then there exist $p$ and $q \in B_{s}$ such that $f(p)=L_{1}$ and $f(q)=L_{2}$. By the assumption (1), $p$ or $q \in V_{1}^{\circ}$ and so, without loss of generality, we may assume $p \in V_{1}^{\circ}$. As $D$ is irreducible, hence we can choose $\left\{q_{1}, q_{2}, \ldots, q_{n+1}\right\} \subset B_{s}$ so that

(i) $q_{1}=p, q_{n+1}=q$,

(ii) $\left\{q_{1}, q_{1}, \ldots, q_{n}\right\} \subset V_{1}^{\circ}$,

(iii) $D_{F_{s}^{-1}\left(q_{i}\right) F_{s}^{-1}\left(q_{i+1}\right)}>0$ for $i=1,2, \ldots, n$.

Then, by the definition of $H_{1}$,

$$
\left(H_{1}\right)_{q_{i} q_{i+1}} \geq D_{F_{s}^{-1}\left(q_{i}\right) F_{s}^{-1}\left(q_{i+1}\right)}>0 .
$$

On the other hand, if $f(r)=L_{1}$ for $r \in V_{1}^{\circ}$, then $f\left(r^{\prime}\right)=L_{1}$ for all $r^{\prime}$ with $\left(H_{1}\right)_{r r^{\prime}} \neq 0$ because

$$
H_{1, r} f=\sum_{r^{\prime}}\left(H_{1}\right)_{r r^{\prime}}\left(f\left(r^{\prime}\right)-f(r)\right)=0 .
$$

Therefore, applying the above discussion inductively, we can show that $f\left(q_{i}\right)=$ $L_{1}$ for $i=1,2, \ldots, n+1$. This contradicts the fact that $f\left(q_{n+1}\right)=f(q)=$ $L_{2}<L_{1}$. Hence we have $v\left(A_{s} f\right)<v(f)$.

Lemma B.3. There exists a constant $C$ with $0<C<1$ such that, for all $f \in l\left(V_{0}\right)$ and $w \in W_{*}, v\left(A_{w} f\right) \leq C^{|w|} v(f)$.

Proof. We define $V=\left\{f \mid f \in l\left(V_{0}\right)\right.$ and $\left.\sum_{i=1}^{M} f_{i}=0\right\}$ and $Q: l\left(V_{0}\right) \rightarrow V$ by, for each $i,(Q f)_{i}=f_{i}-M^{-1} \sum_{i=1}^{M} f_{i}$. Then, it follows that $v(A Q f)=v(A f)$ and $v(f)=v(Q f)$. Hence, we have

$$
\max _{v(f) \neq 0} v\left(A_{s} f\right) / v(f)=\max _{f \in V, v(f)=1} v\left(A_{s} f\right) / v(f) .
$$


Now, as $\{f \mid f \in V, v(f)=1\}$ is compact, using Lemma B.2, we have $C_{s}=$ $\max _{v(f) \neq 0} v\left(A_{s} f\right) / v(f)<1$. Therefore, let $C=\max _{s \in S} C_{s}$, then $0<C<1$ and $v\left(A_{w} f\right) \leq C^{|w|} v(f)$ for all $f \in l\left(V_{0}\right)$ and $w \in W_{*}$.

Next, for given $\rho \in l\left(V_{0}\right)$, we define $f \in l\left(V_{*}\right)$ by, for each $w \in W_{*}$, $f_{w}^{b}=A_{w} \rho$.

Lemma B.4. Let $\left\{p_{n}\right\}_{n=1}^{\infty} \subset V_{*}$ and $p_{n} \rightarrow p$ as $n \rightarrow \infty$ for some $p \in K$. Then $\left\{f\left(p_{i}\right)\right\}_{i=1}^{\infty}$ is convergent as $n \rightarrow \infty$.

Proof. Let $K_{m, p}=\bigcup_{w \in W_{m}, p \in K_{w}} K_{w}$, then $K_{m, p} \supset K_{m+1, p}$ and $K_{m, p}$ is a neighborhood of $p$. Hence, for given $\varepsilon>0$, we can choose $m$ and $n_{0}$ so that $2 C^{m} v(\rho)<\varepsilon$ and, if $n>n_{0}, p_{n} \in K_{m, p}$.

Recalling that $A_{s}$ is a stochastic matrix, we have

$$
\min _{q \in K_{m, p} \cap V_{m}} f(q) \leq f\left(p_{n}\right) \leq \max _{q \in K_{m, p} \cap V_{m}} f(q) .
$$

And so, using Lemma B.3, we can see that, if $k_{1}, k_{2}>n_{0}$, then

$$
\left|f\left(p_{k_{1}}\right)-f\left(p_{k_{2}}\right)\right| \leq 2 \max _{w \in W_{m}, p \in K_{w}} v\left(A_{w} f\right)<\varepsilon .
$$

Hence $\left\{f\left(p_{n}\right)\right\}_{n=1}^{\infty}$ is a Cauchy sequence.

Using the above Lemma, we can extend $f \in l\left(V_{*}\right)$ to a continuous function $f$ on $K$. In fact, if $\left\{p_{n}\right\}_{n=1}^{\infty},\left\{q_{n}\right\}_{n=1}^{\infty} \subset V_{*}$ and both sequences converges to the same point $p \in K$, let

$$
\widetilde{p}_{n}= \begin{cases}p_{m} & \text { if } n=2 m+1 \\ q_{m} & \text { if } n=2 m\end{cases}
$$

Then using Lemma B.4, $\left\{f\left(\tilde{p}_{n}\right)\right\}$ is convergent and so we can show that

$$
\lim _{n \rightarrow \infty} f\left(p_{n}\right)=\lim _{n \rightarrow \infty} f\left(q_{n}\right) .
$$

Thus, this $f$ is the harmonic function with $\left.f\right|_{V_{0}}=\rho$. Uniqueness is obvious because $\left.f\right|_{V_{*}}$ is uniquely determined as is observed in Lemma B.4.

\section{REFERENCES}

1. M. T. Barlow and R. F. Bass, The construction of Brownian motion on the Sierpinski carpet, Ann. Inst. H. Poincaré 25 (1989), 225-257.

2. M. T. Barlow and E. A. Perkins, Brownian motion on the Sierpinski gasket, Probab. Theory Related Fields 79 (1988), 543-624.

3. K. J. Falconer, The geometry of fractal sets, Cambridge, 1985.

4. M. Fukushima, Dirichlet forms and Markov processes, North-Holland/Kodansha, 1980.

5. M. Hata, On the structure of self-similar sets, Japan J. Appl. Math. 2 (1985), 381-414.

6. M. Hata and M. Yamaguti, The Takagi function and its generalization, Japan J. Appl. Math. 1 (1984), 183-199.

7. K. Hattori, T. Hattori, and H. Watanabe, Gaussian field theories on general networks and the spectral dimensions, Progr. Theoret. Phys. Suppl. 92 (1987), 108-143.

8. J. E. Hutchinson, Fractals and self-similarity, Indiana Univ. Math. J. 30 (1981), 713-747.

9. A. Kameyama, Kyoto Univ. Master Thesis, 1989.

10. J. Kigami, A harmonic calculus on the Sierpinski spaces, Japan J. Appl. Math. 6 (1989), 259-290. 
11. S. Kusuoka, A diffusion process on a fractal, Probabilistic Methods in Mathematical Physics, Proc. Taniguchi Internat. Sympos. (Katata and Kyoio, 1985), (K. Ito and N. Ikeda, eds.), Kinokuniya, Tokyo, 1987, pp. 251-274.

12. _ Dirichlet forms on fractals and products of random matrices, Publ. Res. Inst. Math. Sci. 25 (1989), 659-680.

13. T. Lindstrøm, Brownian motion on nested fractals, Mem. Amer. Math. Soc. no. 420 (1990).

14. T. Shima, On eigenvalue problems for the random walks on the Sierpinski pre-gaskets, Japan J. Indust. Appl. Math. 8 (1991), 124-141.

15. M. Yamaguti and J. Kigami, Some remarks on Dirichlet problem of Poisson equation, Analyse Mathématique et Application, Gauthier-Villars, Paris, 1988, pp. 465-471.

16. M. Fukushima and T. Shima, On a spectral analysis for the Sierpinski gasket, Preprint (1989).

Department of Mathematics, College of General Education, Osaka University, ToyONAKA 560, JAPAN

Current address: Department of Mathematics, University of California, Riverside, California 92507

E-mail address: jkigami@ucrmath.ucr.edu 